\title{
X-RAY-EMITTING STARS IDENTIFIED FROM THE ROSAT ALL-SKY SURVEY AND THE SLOAN DIGITAL SKY SURVEY*
}

\author{
Marcel A. Agüeros ${ }^{1,7}$, Scott F. Anderson ${ }^{2}$, Kevin R. Covey $^{3,8}$, Suzanne L. Hawley $^{2}$, Bruce Margon $^{4}$, \\ Emily R. Newsom ${ }^{1}$, Bettina Posselt ${ }^{5,9}$, Nicole M. Silvestri ${ }^{2}$, Paula Szkody ${ }^{2}$, and Wolfgang Voges ${ }^{6}$ \\ ${ }^{1}$ Columbia Astrophysics Laboratory, Columbia University, 550 West 120th Street, New York, NY 10027, USA; marcel@astro.columbia.edu \\ ${ }^{2}$ Department of Astronomy, University of Washington, Box 351580, Seattle, WA 98195, USA \\ ${ }^{3}$ Harvard-Smithsonian Center for Astrophysics, 60 Garden Street, Cambridge, MA 02138, USA \\ ${ }^{4}$ Department of Astronomy and Astrophysics, University of California, 1156 High Street, Santa Cruz, CA 95064, USA \\ ${ }^{5}$ Harvard-Smithsonian Center for Astrophysics, 60 Garden Street, Cambridge, MA 02138, USA \\ ${ }^{6}$ Max-Planck-Institut für extraterrestrische Physik, Geissenbachstrasse 1, D-85741 Garching, Germany \\ Received 2007 July 25; accepted 2009 January 14; published 2009 March 19
}

\begin{abstract}
The ROSAT All-Sky Survey (RASS) was the first imaging X-ray survey of the entire sky. Combining the RASS Bright and Faint Source Catalogs yields an average of about three X-ray sources per square degree. However, while $\mathrm{X}$-ray source counterparts are known to range from distant quasars to nearby M dwarfs, the RASS data alone are often insufficient to determine the nature of an X-ray source. As a result, large-scale follow-up programs are required to construct samples of known X-ray emitters. We use optical data produced by the Sloan Digital Sky Survey (SDSS) to identify 709 stellar X-ray emitters cataloged in the RASS and falling within the SDSS Data Release 1 footprint. Most of these are bright stars with coronal X-ray emission unsuitable for SDSS spectroscopy, which is designed for fainter objects $(g>15$ [mag]). Instead, we use SDSS photometry, correlations with the Two Micron All Sky Survey and other catalogs, and spectroscopy from the Apache Point Observatory $3.5 \mathrm{~m}$ telescope to identify these stellar Xray counterparts. Our sample of 707 X-ray-emitting F, G, K, and M stars is one of the largest X-ray-selected samples of such stars. We derive distances to these stars using photometric parallax relations appropriate for dwarfs on the main sequence, and use these distances to calculate $L_{X}$. We also identify a previously unknown cataclysmic variable (CV) as a RASS counterpart. Separately, we use correlations of the RASS and the SDSS spectroscopic catalogs of CVs and white dwarfs (WDs) to study the properties of these rarer X-ray-emitting stars. We examine the relationship between $\left(f_{X} / f_{g}\right)$ and the equivalent width of the $\mathrm{H} \beta$ emission line for $46 \mathrm{X}$-ray-emitting CVs and discuss tentative classifications for a subset based on these quantities. We identify 17 new X-ray-emitting DA (hydrogen) WDs, of which three are newly identified WDs. We report on follow-up observations of three candidate cool X-rayemitting WDs (one DA and two DB (helium) WDs); we have not confirmed X-ray emission from these WDs.
\end{abstract}

Key words: surveys - X-rays: stars

Online-only material: color figures, machine-readable table

\section{INTRODUCTION}

X-ray data obtained since the 1970s have shown definitively that stellar X-ray emitters are present among almost all stellar classes (e.g., Harnden et al. 1979; Cassinelli \& Swank 1983; Stocke et al. 1983; Schmitt et al. 1995; Motch et al. 1998; Zickgraf et al. 2003; Rogel et al. 2006), with the emission mechanism being different for early and late-type stars; only evolved $\mathrm{K}$ and $\mathrm{M}$ stars and perhaps A stars do not appear to be X-ray emitters (e.g., Ayres et al. 1981; Antiochos et al. 1986; Haisch et al. 1991; Daniel et al. 2002). However, within a stellar class - particularly among late-type stars-the strength of the $\mathrm{X}$-ray emission varies greatly. A G5 star may be much brighter in the X-ray regime than its apparent twin, suggesting that the physics of X-ray emission is intimately linked to detailed stellar properties. X-ray spectroscopy with the Chandra X-ray Observatory and XMM-Newton X-ray Observatory, occasionally in conjunction with observations at other wavelengths, is providing unprecedented insight into these connections (e.g., Osten

\footnotetext{
* Includes observations obtained with the Apache Point Observatory $3.5 \mathrm{~m}$ telescope, which is owned and operated by the Astrophysical Research Consortium.

7 NSF Astronomy and Astrophysics Postdoctoral Fellow.

8 Spitzer Fellow.

9 Leopoldina Fellow.
}

et al. 2005; Smith et al. 2005; Hussain et al. 2007). Before such studies can be conducted, however, catalogs of confirmed $\mathrm{X}$-ray-emitting stars from all areas of the Hertzsprung-Russell diagram need to be compiled and broadly characterized.

We have identified a sample of stellar X-ray emitters cataloged in the ROSAT All-Sky Survey (RASS; Voges et al. 1999) and falling within the footprint of the Sloan Digital Sky Survey (SDSS; York et al. 2000) Data Release 1 (DR1; Abazajian et al. 2003). We produced a two-part catalog. One part includes 231 stars that are previously cataloged, e.g., in SIMBAD or in the NASA/IPAC Extragalactic Database (NED), ${ }^{10}$ but not identified as X-ray source counterparts. Another 478 entries are new stellar $\mathrm{X}$-ray source identifications made on the basis of spectroscopic observations with the Astrophysical Research Consortium $3.5 \mathrm{~m}$ telescope at the Apache Point Observatory (APO). The vast majority of these stars are F, G, K, and M stars (707 stars), although we identify a new cataclysmic variable (CV) in our APO data; the SIMBAD sample also includes a newly identified X-ray-emitting white dwarf (WD). To expand the sample

\footnotetext{
10 This research has made use of the SIMBAD database, which is operated at CDS, Strasbourg, France, and of the NASA/IPAC Extragalactic Database and Infrared Science Archive, which are operated by the Jet Propulsion Laboratory, California Institute of Technology, under contract with the National Aeronautics and Space Administration.
} 
of X-ray-emitting CVs and WDs, we correlated the SDSS spectroscopic catalogs of these objects (Szkody et al. 2002, 2003, 2004a, 2005, 2006, 2007; Eisenstein et al. 2006) with the RASS to identify the X-ray emitters among these more exotic stars. In a previous paper, we described our efforts to use the RASS and SDSS to identify new isolated neutron stars, which are possibly the rarest stellar X-ray emitters (Agüeros et al. 2006).

In Section 2, we review other efforts to construct large samples of X-ray-emitting stars; we also provide a brief description of SDSS, as well as a summary of the challenges of using SDSS to identify RASS stellar X-ray emitters. In Section 3, we outline the process by which we constructed our list of candidate DR1 stellar counterparts to RASS sources. In Section 4, we describe how we confirmed our stellar identifications, thereby obtaining our sample of stellar X-ray sources. We distinguish between previously cataloged stars we identify as X-ray sources and stars for which we obtained spectra with the APO $3.5 \mathrm{~m}$ telescope. In Section 5, we begin by calculating distances and $\mathrm{X}$-ray luminosities for 662 stars in our catalog, compare our identifications to those of Flesch \& Hardcastle (2004) and of Parejko et al. (2008) for sources that also appear in their catalogs, and describe the data in our main catalog, which includes the X-ray, optical, and infrared information for our 709 stellar X-ray sources. We also estimate the overall reliability of our identifications; we find that $\lesssim 10 \%$ are likely to be false associations. In Section 6, we present the results of a parallel effort to use correlations of the SDSS spectroscopic catalogs of CVs and of WDs with the RASS to construct catalogs of these rarer stellar $\mathrm{X}$-ray emitters. We also report on follow-up Chandra and XMM observations of several candidate cool X-ray-emitting WDs. We conclude in Section 7.

\section{IDENTIFYING STELLAR X-RAY SOURCES}

\subsection{Using Optical Photometry and Spectroscopy}

The Einstein Observatory provided the first large samples of $\mathrm{X}$-ray sources. The Medium Sensitivity Survey (MSS; Gioia et al. 1984) and the Extended Medium-Sensitivity Survey (EMSS; Gioia et al. 1990) collected serendipitously detected sources with fluxes between $\sim 5 \times 10^{-14}$ and $3 \times 10^{-12} \mathrm{erg}$ $\mathrm{cm}^{-2} \mathrm{~s}^{-1}$ in the $0.3-3.5 \mathrm{keV}$ energy band. Because survey X-ray data are often insufficient to determine unambiguously the nature of a source, extensive optical follow-up was required to identify the 112 MSS and the 835 EMSS sources. Stocke et al. (1983) used the MSS to confirm that a given class of X-ray emitters has a fairly narrow range of possible X-ray-to-optical flux ratios (e.g., for active galactic nuclei (AGNs), $-1.0 \leqslant \log$ $\left.\left(f_{X} / f_{V}\right) \leqslant+1.2\right)$, and that the overlap between broad classes (e.g., Galactic and extragalactic sources) is fairly small. They found that $\sim 25 \%$ of MSS sources were coronally emitting stars, primarily late-type dwarfs; they also found one CV. Stocke et al. (1991) used the MSS results to identify plausible counterparts to EMSS sources based on their $\left(f_{X} / f_{\text {opt }}\right)$. Still, confirming that these counterparts were $\mathrm{X}$-ray sources was very time intensive: Stocke et al. (1991) obtained two to five spectra in each Xray error circle. Six to eight nights a year over seven years were needed at the Multiple Mirror Telescope Observatory, Mt. Hopkins, AZ, to identify 665 sources. This work confirmed that $\sim 25 \%$ of Einstein detected sources are Galactic stars.

Similar efforts have been undertaken to identify some of the $\sim 125,000$ sources detected at soft X-ray energies $(0.1-2.4 \mathrm{keV})$ by ROSAT as part of its All-Sky Survey. The RASS Bright Source Catalog (BSC; Voges et al. 1999) includes 18, 811 sources with count rates $\geqslant 0.05$ counts $\mathrm{s}^{-1}$ and $\geqslant 15$ counts; the Faint Source Catalog (FSC; Voges et al. 2000) lists another 105,924 with $<0.05$ counts $\mathrm{s}^{-1}$. A relatively small fraction of RASS sources can be identified from correlations with existing catalogs. Bade et al. (1998) found matches to $35 \%$ of a sample of 80,000 RASS sources in SIMBAD and NED. To identify other BSC sources, Bade et al. (1998) used objective prism spectra obtained during the Hamburg Quasar Survey (HQS; Hagen et al. 1995 ) and found candidate counterparts for $81.2 \%$ of the 3847 sources within the HQS footprint. Of these, 155 (4\%) were M stars, $136(3.5 \%) \mathrm{K}$ stars, $4(0.1 \%) \mathrm{F}$ or G stars; a further 956 (24.9\%) were saturated bright stars $(B \leqslant 14 \mathrm{mag})$, for which no spectral class is available. There were also $31 \mathrm{WDs}(0.8 \%)$ and $16 \mathrm{CVs}(0.4 \%)$. There are uncertainties associated with these identifications; the spectra are fairly low resolution, for example $(R \approx 100$ at $\mathrm{H} \gamma)$. But the RASS/HQS program suggests that one-third of ROSAT-detected sources are Galactic stars.

Zickgraf et al. (2003) applied this method to identify all 5341 RASS BSC sources with $\delta \geqslant 0^{\circ}$ and Galactic latitudes $|b| \geqslant 30^{\circ}$; the total area covered was $10,313 \mathrm{deg}^{2}$. Candidate counterparts were found for $82.2 \%$ of the RASS sources; the "missing" counterparts are faint optical objects whose poorquality prism spectra preclude classification. The fraction of the 5341 BSC sources due to coronal emission from stars increases from $33 \%$ to $\sim 38 \%$ when one includes the likely stellar sources among the missing counterparts (Zickgraf et al. 2003). The difference between this fraction and that of stellar sources in the EMSS may be due to the different energy sensitivities of ROSAT and Einstein (Zickgraf et al. 2003). The final RASS/ HQS sample includes $197 \mathrm{M}$ stars, $141 \mathrm{~K}$ stars, $45 \mathrm{~F} / \mathrm{G}$ stars, and 1219 saturated stars without spectral classifications, along with 45 WDs and 26 CVs.

\subsection{Using Catalog Matching}

Parallel efforts have been made to produce large-scale catalogs of RASS counterparts without spectroscopy. Voges et al. (1999) correlated the 18, 811 BSC sources with a number of existing catalogs_-for example, of bright stars (Tycho; Høg et al. 1998) or of radio and ultraviolet (UV) sources (FIRST, NVSS, and EUVE; Becker et al. 1995; Condon et al. 1998; Bowyer et al. 1996). They found at least one cataloged counterpart within $90^{\prime \prime}$ for $90 \%$ of BSC sources. 7117 X-ray sources had a single cataloged counterpart, which Voges et al. (1999) used to establish the overall statistical properties of the catalog: 58\% have Galactic counterparts. This matching was based primarily on positional coincidence and relied on the relatively low surface densities of BSC sources and of the various candidate counterparts (Voges et al. 1999).

Rutledge et al. (2000) matched the BSC to the USNO A-2 catalog (Monet et al. 1998). Factoring in both the proximity and the USNO $B$ of objects found within $75^{\prime \prime}$ of the RASS sources, and estimating the contamination rate by comparisons to background fields, they sifted through $>320,000$ possible optical counterparts to identify those with the highest probability of being X-ray sources. The resulting catalog includes 11, 301 BSC sources for which Rutledge et al. (2000) identified a USNO object with a $\geqslant 50 \%$ probability of being the counterpart; 2705 had a probability $\geqslant 98 \%$.

In an even more ambitious effort, Flesch \& Hardcastle (2004) matched X-ray data from all of the major ROSAT catalogs to multiple radio and optical surveys, thereby creating an allsky catalog of radio/optical counterparts to some 500,000 Xray sources. Nearly 120,000 of these sources were previously 
identified, while additional 86,000 are estimated to be 40\%-99\% likely to be previously unknown quasars (quasi-stellar objects (QSOs)). The likelihood of a given association was calculated first by examining the proximity of a proposed counterpart, its $B-R$ color, and its optical classification, and then by comparing the density of all candidate counterparts with these properties to their all-sky density (Flesch \& Hardcastle 2004).

These catalogs are very useful for developing a statistical sample of a given category of X-ray emitters, typically QSOs (e.g, Bauer et al. 2000; Flesch \& Hardcastle 2004). (There is usually almost no discussion of the stellar X-ray sources.) However, because they rely largely on the properties of known counterparts to identify new counterparts, the catalogs cannot tell us more about the overall properties of X-ray sources than the smaller catalogs on which they are based (Flesch \& Hardcastle 2004). In that sense, these catalog-based approaches cannot fully replace the combined photometric and spectroscopic method developed to identify Einstein sources.

Furthermore, detailed analysis of selected subsets of these catalogs can reveal problems with the claimed identifications. For example, intrigued by apparent anomalies in the spatial distribution of QSOs in the Flesch \& Hardcastle (2004) catalog, López-Corredoira et al. (2008) investigated a subsample of 41 QSO candidates to test the reliability of their assigned QSO likelihoods. They found that only 12 were actual QSOs, and that the catalog likelihoods were particularly unreliable for bright objects ( $B \leqslant 15.1 \mathrm{mag}$ ), where none of the 13 Flesch \& Hardcastle (2004) candidates was in fact a QSO.

\subsection{Using SDSS}

Previous to this work, the Zickgraf et al. (2003) catalog was the single largest sample of RASS stellar sources for which counterparts have been spectroscopically confirmed. In fact, only $\sim 10 \%$ of RASS BSC/FSC sources are identified and fully characterized (McGlynn et al. 2004; Mickaelian et al. 2006), with the majority of the identifications being of BSC sources. Large-scale programs to find the counterparts to RASS sources are still very much needed.

SDSS provides uniform optical photometric and spectroscopic data with which to correlate the RASS catalogs. The survey produces $u, g, r, i, z$ images to a depth of $r \sim 22.5 \mathrm{mag}$ (Fukugita et al. 1996; Gunn et al. 1998; Hogg et al. 2001; Smith et al. 2002; Gunn et al. 2006), with a photometric accuracy of $\sim 0.02$ mag (Ivezić et al. 2004); Tucker et al. (2006) describe the photometric pipeline. The imaging survey covers $8420 \mathrm{deg}^{2}$ and includes photometric data for over $10^{8}$ stars and a similar number of galaxies. SDSS is also a spectroscopic survey, and has obtained spectra for $\sim 8 \times 10^{5}$ galaxies, $\sim 10^{5}$ QSOs, and $\sim 2 \times 10^{5}$ stars.

SDSS is an ideal tool for systematically identifying large numbers of ROSAT sources (e.g., X-ray-emitting AGN or galaxies; Anderson et al. 2003, 2007; Parejko et al. 2008). However, the vast majority of stellar X-ray emitters cataloged in the RASS are unlikely to be discovered from routine SDSS spectroscopy. Relative to their optical output, the most X-ray luminous normal stars (i.e., coronal emitters) have $\log \left(f_{X} / f_{\text {opt }}\right)$ $\sim-1$ (Maccacaro et al. 1988) and so almost all are optically brighter than the $g \approx 15$ mag SDSS spectroscopic limit.

We have therefore combined the SDSS photometric data with infrared data from the Two Micron All Sky Survey (2MASS; Skrutskie et al. 2006) to select a sample of bright stars near BSC/FSC positions. When available, we used existing catalog data to classify the stars. In many cases, however, no spectral class is available, and we therefore used the APO $3.5 \mathrm{~m}$ telescope to obtain spectra for objects in about 900 of these RASS error circles (the total number of spectra collected is close to 1200). We then used a spectral-template fitting code to classify the likely counterparts by spectral type. We also estimated $\left(f_{X} / f_{\text {opt }}\right)$ ratios for all the stars based on their cataloged magnitudes and compared the ratios to those of known X-ray emitters of the same spectral type, thereby verifying that these stars are indeed plausible RASS source counterparts. Each of these steps is described in detail in the following sections.

\section{SELECTING X-RAY-EMITTING STARS FROM THE RASS AND SDSS DR1}

We matched the $\sim 125,000$ RASS X-ray source positions to those of the SDSS DR1 (Abazajian et al. 2003) stripes; each SDSS stripe is a $2.5 \mathrm{deg}$ wide rectangle of sky. We found matches for 7464 RASS sources, or just under $6 \%$ of the catalogs' entries. Since the DR1 area is $2099 \mathrm{deg}^{2}$ (Abazajian et al. 2003), or roughly $5 \%$ of the entire sky, this number is consistent with a naive surface density expectation. ${ }^{11}$

We queried the SDSS photometric database using the SDSS Query Analyzer (sdssQA) to identify bright stars near these RASS sources; see Agüeros (2006) for details. This query returned coordinates, PSF magnitudes, ${ }^{12}$ and automatically assigned morphology type (star or galaxy) for objects with $g$ or $r<16$ mag, subject to a small number of tests of the objects' photometric flags.

The output from sdssQA was matched to the RASS sources in the DR1 footprint. This matching produced a list of $\lesssim 5000$ RASS sources with a bright/saturated SDSS object within 1', roughly two-thirds of the total number of RASS/DR1 sources. However, visual inspection of the SDSS images for these sources revealed that a significant fraction did not have a nearby bright object. ${ }^{13}$ While a more conservative set of photometric cuts might eliminate many contaminants, it might also remove the real but saturated objects in which we are interested. We therefore used the Two Micron All Sky Survey (2MASS; Skrutskie et al. 2006) to confirm the existence of bright SDSS objects in the RASS error circles. While SDSS is a much deeper survey than 2 MASS, whose $J, H$, and $K_{s}$ limits are $\sim 14$ mag, the two surveys are well matched at the bright end $(r \leqslant 16)$, and our target sample of bright SDSS objects should be detected by 2MASS.

Accordingly, the 2MASS catalog was queried for matches to the 5000 SDSS objects. The typical positional uncertainties for point sources in both surveys are on the order of a fraction of an arcsecond (e.g., Finlator et al. 2000; Pier et al. 2003); the median separation between matches was 0.22 . This matching left us with a list of 2520 bright/saturated SDSS objects with a nearby 2MASS counterpart within $1^{\prime}$ of a RASS source. In Figure 1, we show the normalized histogram of the resulting RASS/SDSS separations $\leqslant 30^{\prime \prime}$.

To estimate the likelihood of false matches, we shifted the positions of RASS sources in the DR1 footprint by $2^{\prime}$ in declination and searched for nearby SDSS objects with 2MASS counterparts in the same manner as described above. Figure 1

\footnotetext{
11 The quoted DR1 area is for the imaging survey; the DR1 spectroscopic area is $1360 \mathrm{deg}^{2}$.

12 PSF fitting provides better estimates of isolated star magnitudes; see Stoughton et al. (2002).

13 We used the SDSS image list tool to examine our error circles (Nieto-Santisteban et al. 2004).
} 


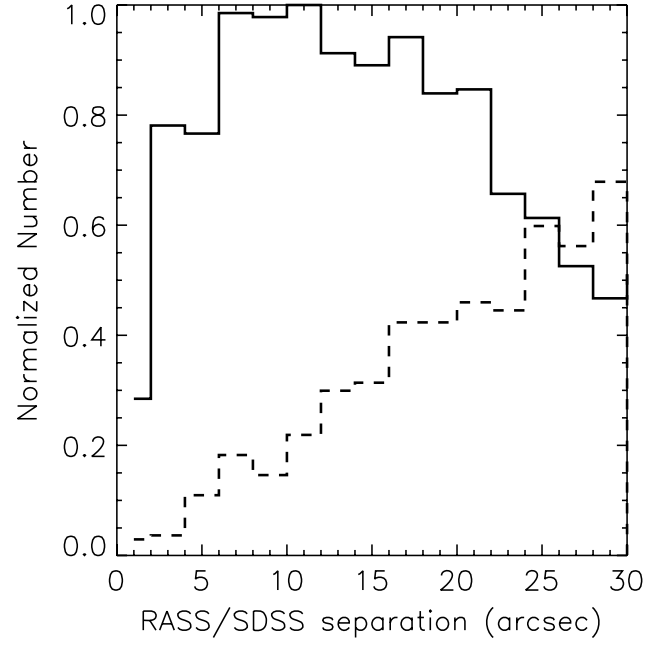

Figure 1. Solid line: distribution of separations between RASS sources and nearby bright/saturated SDSS objects with 2MASS counterparts. Dashed line: distribution of separations returned by matching shifted X-ray sources to SDSS/ 2MASS objects.

shows the (dashed) normalized histogram for this control sample. The number of false matches rises with separation, as expected. The normalization is the same as for the previous matches, so that the dashed histogram gives an upper limit to the contamination of our sample by chance superpositions of independent RASS sources and optical/IR objects. At 25", the cumulative contamination is $\sim 35 \%$. Absent other information, e.g., the brightness or nature of the proposed counterpart, positional proximity alone is generally insufficient to make secure RASS source identifications from SDSS/2MASS data. We return to the question of contamination by false matches in Section 5.4, after we have assembled our final catalog.

In general, the SDSS spectroscopic database does not provide the needed additional information to identify stellar RASS counterparts. As objects brighter than $\sim 15$ mag are rarely targeted, the RASS counterparts most likely to be targeted for spectroscopy are QSOs and other AGN, since they tend to be optically fainter than X-ray-emitting stars (see Anderson et al. 2003, 2007). In 2003 September, 680,000 SDSS spectra were available, a sample we defined as making up the DR1 spectroscopic survey, yet only $\sim 70$ of our matched RASS/SDSS/2MASS objects have an associated SDSS spectrum. Clearly, the majority of our RASS DR1 sources cannot therefore be directly identified from SDSS spectroscopy.

\section{THE RASS/SDSS DR1 CATALOG OF STELLAR X-RAY SOURCES}

To identify new stellar X-ray sources, we begin by searching SIMBAD to find stars with known spectral types recovered in our RASS/SDSS correlation. Among these are a subset of previously identified X-ray sources, to which we add the (optically bright) cataloged stars with small offsets relative to the RASS positions to construct flux ratio distributions. Using spectral types obtained from our APO observations, we then find those previously uncataloged stars whose $\log \left(f_{X} / f_{J}\right)$ falls within the $2 \sigma$ range obtained from our RASS/SIMBAD sample. Our final catalog includes these stars along with those cataloged in SIMBAD but not previously identified as RASS sources. The steps in constructing this catalog are described in detail below.

\subsection{RASS/SIMBAD Stars}

We searched SIMBAD for cataloged candidate stellar counterparts to our 2520 matched RASS sources and found 457 stars with an associated spectral type. Examining the literature, we determined that 159 of these stars were previously associated with ROSAT X-ray sources (e.g., by Dempsey et al. 1993; Appenzeller et al. 1998; Fleming 1998; Hünsch et al. 1998a, 1998b; Metanomski et al. 1998; Hünsch et al. 1999; Schwope et al. 2000; Zickgraf et al. 2003) and more rarely with sources detected by Einstein (e.g., by Topka et al. 1982; Fleming et al. 1989a, 1989b; Stocke et al. 1991; Drake et al. 1992) or by an even earlier X-ray mission (e.g., the RS CVn systems DM UMa, detected by the High Energy Astrophysics Observatory-1 and IN Vir, by EXOSAT; Walter et al. 1980; Giommi et al. 1991). In four additional cases the stars were listed as the RASS source counterparts, but the origin of the association was unclear. ${ }^{14}$ Conversely, 294 of these SIMBAD stars had not been associated with an X-ray source.

Unsurprisingly, these stars tend to be optically very bright. 427 have a cataloged $V$; for these, the median is 8.95 mag. The stars are generally associated with brighter X-ray sources: the sample median count rate is 0.04 counts $\mathrm{s}^{-1}$. By contrast, the median for the 2520 RASS sources is 0.03 counts $s^{-1}$. The median separation between RASS sources and SIMBAD stars is 12 . 0 ; this is equivalent to $0.9 \sigma$, where $\sigma$ is the separation between the matched positions divided by the RASS positional uncertainty (see Figure 2). The offset distribution of the known $\mathrm{X}$-ray emitters in the sample suggests that the number of $\mathrm{X}$-ray source counterparts drops quickly for separations $\gtrsim 30^{\prime \prime}$ or $\gtrsim 2 \sigma$; only eight stars are at offsets $>30^{\prime \prime}$; six have separations $>2 \sigma$.

The optical brightness and small positional offsets of the previously unpublished RASS/SIMBAD matches strongly suggest that they are also the X-ray sources. We replicate the matching to the RASS BSC done by Voges et al. (1999) for stars in the Tycho catalog; the matched stars are comparable in brightness to our SIMBAD stars (their median $V \sim 10 \mathrm{mag}$ ). For offsets $<30^{\prime \prime}$ the fraction of chance Tycho/RASS coincidences is $\sim 2 \%$. This implies that $\sim 248$ of the 253 stars with offsets $<30^{\prime \prime}$ not yet associated with an X-ray source are likely to be RASS source counterparts.

Figure 3 displays the RASS count rate versus SIMBAD $V$ for 139 known X-ray emitters and for 249 other SIMBAD stars with $<30^{\prime \prime}$ offsets. ${ }^{15}$ These are primarily F, G, K, and M stars, although there are a few candidate A star counterparts (see Appendix A). There are also a handful of WDs (including a WD/M-star binary) and of CVs, which stand out because of their relative optical faintness but $\mathrm{X}$-ray brightness. The brightest source is the low-mass X-ray binary Hercules X-1, among the first extrasolar X-ray sources to be detected (Schreier et al. 1972; Tananbaum et al. 1972).

\subsubsection{X-Ray-to-Optical Flux Ratio Calculations}

Numerous studies have shown that there is a typical range of X-ray-to-optical flux ratios for each stellar type (e.g., Stocke et al. 1991; Krautter et al. 1999; Zickgraf et al. 2003). Accordingly, we calculate $\log \left(f_{X} / f_{V}\right)$ for our stars. We exclude the 23 stars listed in SIMBAD as belonging to luminosity classes II,

\footnotetext{
14 These are FBS 0124-098, NLTT 6782, FBS 0249-084, and TYC $255-263-1$.

1520 known X-ray-emitting stars lack a cataloged $V$, as do four previously unidentified candidate RASS counterparts.
} 

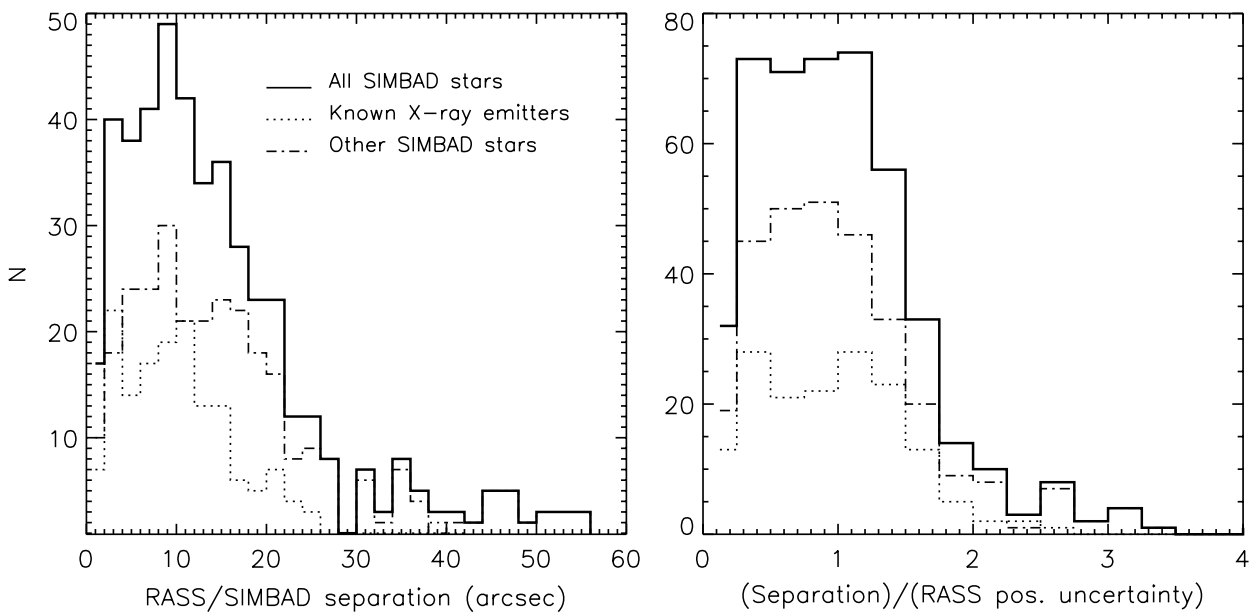

Figure 2. Left panel: distribution of positional offsets between the RASS sources and the SIMBAD stars. Right panel: the same distribution, expressed in terms of the cataloged X-ray positional uncertainty.

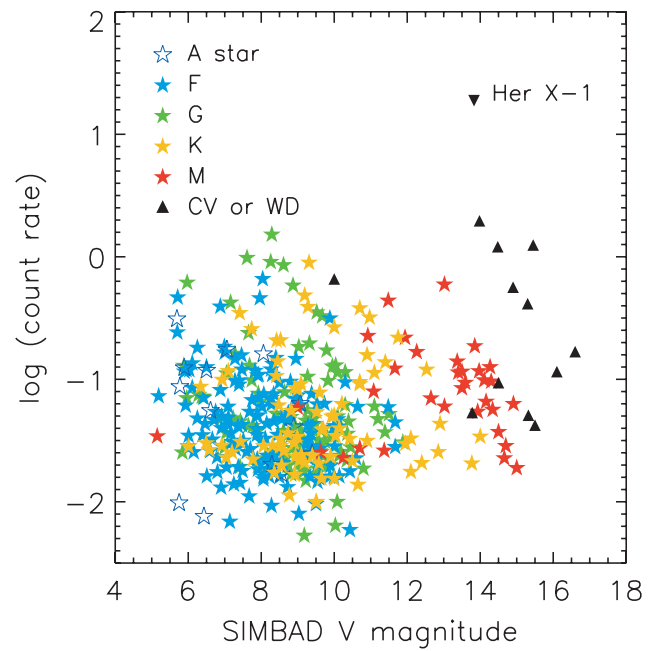

Figure 3. X-ray count rate vs. $V$ for 139 known $X$-ray-emitting SIMBAD stars and 249 SIMBAD stars offset by $<30^{\prime \prime}$ from a RASS source. $B$ was used for the two CVs and two WDs with no cataloged $V$.

(A color version of this figure is available in the online journal.)

III, or IV (see Appendix A). We also remove all stars for which the RASS match has an X-ray flag set to 1, which indicates that the X-ray data are not reliable (see Voges et al. 1999).

We do not remove the known binary systems from this sample when calculating the flux ratios. There are 64 such systems within $30^{\prime \prime}$ of a RASS source and with RASS flags set to 0; one more is a known X-ray emitter with an offset $>30^{\prime \prime} .40$ are listed in SIMBAD as double or multiple star systems; one is in a star cluster, and one was identified as a binary through highresolution spectroscopy by Metanomski et al. (1998). Seven of these systems have been previously cataloged as X-ray sources. The sample also includes two Algol-type eclipsing and five spectroscopic binaries, four $\beta$ Lyr, six RS CVn, and six W UMa systems (including W UMa itself). While these systems may seem unrepresentative, many are known X-ray emitters (e.g., all the W UMa and RS CVn systems, but also the Algoltype binaries), which argues for their inclusion in flux ratio calculations. $^{16}$

As part of the spectroscopic campaign described in Section 4.2, we obtained spectra for 40 of the SIMBAD stars

\footnotetext{
16 In practice, including the binaries broadens the flux ratio distributions slightly by adding objects at higher $\left(f_{X} / f_{V}\right)$.
}

with listed spectral types. Only 20 meet the criteria for inclusion in these calculations, and in only three cases would using our APO-based spectral classifications affect the $\left(f_{X} / f_{V}\right)$ distributions. In two cases, the difference in spectral type is consistent with our spectral typing uncertainty (see Section 4.2): V* V842 Her, for which SIMBAD gives an F9V spectral type, and which we identify as a G2 star, and StKM 1-1262, which Stephenson (1986) classifies as a K5 star, and which we find is an M0. In both cases, we use the SIMBAD type. The third star is RX J1057.1-0101, classified as a K4V star by Zickgraf et al. (2005). We type the RASS counterpart as a G5 star; however, examination of this error circle reveals that our APO target is not RX J1057.1-0101 but a nearby star. See Appendix B for further discussion of these stars.

We then have 105 known X-ray-emitting F, G, K, and M stars with a cataloged $V$ and with RASS flags $=0$, and an additional 229 SIMBAD stars not previously associated with an X-ray source that meet the same criteria and are offset by $<30^{\prime \prime}$ from a RASS source. Following Maccacaro et al. (1988), we calculate $\log \left(f_{X} / f_{V}\right)=\log \left(f_{X}\right)+0.4 V+5.37$ and assume that on average 1 count s${ }^{-1}$ translates to $f_{X}=10^{-11} \mathrm{erg} \mathrm{cm}^{-2} \mathrm{~s}^{-1}$ in the 0.1-2.4 keV energy range (e.g., Motch et al. 1998; Voges et al. 1999).

We first create separate distributions for the 105 known X-ray emitters and for the other 229 SIMBAD stars. KolmogorovSmirnov tests of the resulting flux ratio distributions find that for $\mathrm{F}, \mathrm{G}$, and $\mathrm{K}$ stars the $\log \left(f_{X} / f_{V}\right)$ distributions are drawn from different underlying distributions $(P \approx 0)$, while for the M stars the distributions are weakly related $(P=0.6$; however, there are only six previously unidentified candidate $\mathrm{M}$ star counterparts). This is consistent with the fact that the known X-ray emitters are generally somewhat brighter in the optical and significantly brighter in the $\mathrm{X}$ ray than the SIMBAD stars not previously associated with a RASS source. Given the high probability that based on positional coincidence the 229 previously unassociated stars considered here are RASS sources, we group the known and previously unknown X-ray emitters together, and calculate $\log \left(f_{X} / f_{V}\right)$ for all of the stars of a given spectral type. The resulting distributions are presented in Figure 4, as are empirical $2 \sigma$ ranges estimated from the mean $\log \left(f_{X} / f_{V}\right)$ for each spectral type.

We also show in Figure 4 the flux ratio limits for each class as observed by Krautter et al. (1999); they identified 274 stellar X-ray emitters in an area-limited survey of RASS BSC and FSC 

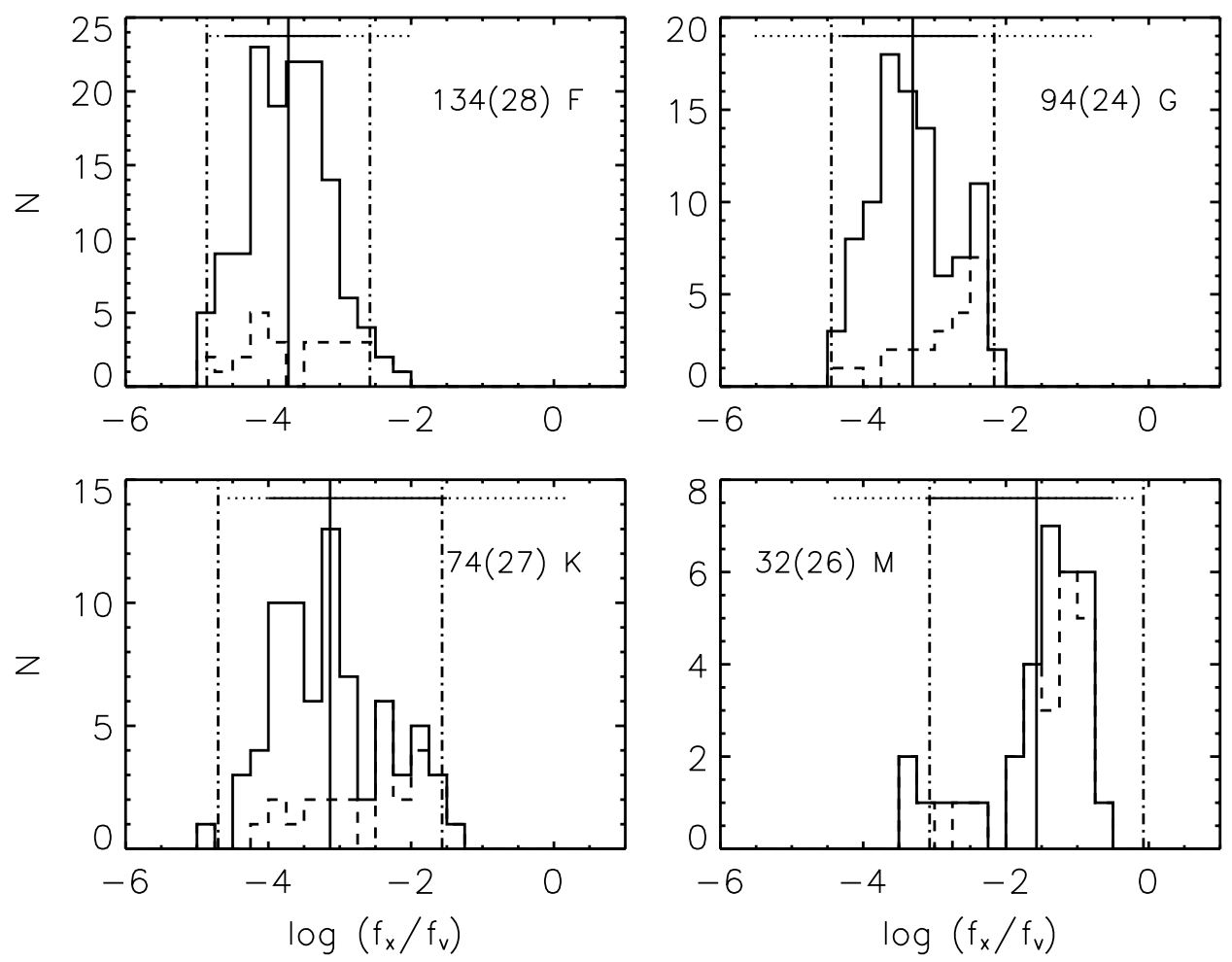

Figure 4. $\log \left(f_{X} / f_{V}\right)$ distributions for the RASS /SIMBAD F, G, K, and M stars. The dashed histograms are the distributions for the subsets of previously known X-ray emitters. The solid vertical lines correspond to the mean $\left(f_{X} / f_{V}\right)$ and the dot-dashed vertical lines bracket the $2 \sigma\left(f_{X} / f_{V}\right)$ range for each stellar type. The number of RASS /SIMBAD stars used to calculate these $\left(f_{X} / f_{V}\right)$ ranges is indicated in each panel; the number in parentheses is the subset of previously known X-ray emitters. The horizontal lines are $\left(f_{X} / f_{V}\right)$ ranges from the literature: the solid lines are from the Maccacaro et al. (1988) Einstein survey, while the dotted extensions are from Krautter et al. (1999).

Table 1

RASS/SIMBAD Stars

\begin{tabular}{|c|c|c|c|c|c|c|c|c|}
\hline \multirow[b]{2}{*}{ Type } & \multirow{2}{*}{$\begin{array}{c}\text { Initial } \\
\text { Number }\end{array}$} & \multirow{2}{*}{$\begin{array}{c}\text { New RASS } \\
\text { Sources }\end{array}$} & \multicolumn{3}{|c|}{$\log \left(f_{X} / f_{V}\right)$} & \multicolumn{3}{|c|}{$\log \left(f_{X} / f_{J}\right)$} \\
\hline & & & Mean & $2 \sigma$ Min & $2 \sigma \mathrm{Max}$ & Mean & $2 \sigma$ Min & $2 \sigma$ Max \\
\hline $\mathrm{F}$ & $169(38)$ & 104 & -3.72 & -4.86 & -2.58 & -3.14 & -4.18 & -2.09 \\
\hline G & 108 (29) & 70 & -3.31 & -4.45 & -2.17 & -2.82 & -4.15 & -1.49 \\
\hline K & $104(42)$ & 47 & -3.13 & -4.70 & -1.57 & -2.77 & -4.27 & -1.27 \\
\hline \multirow[t]{2}{*}{ M } & $45(31)$ & $9^{a}$ & -1.57 & -3.07 & -0.07 & -2.22 & -3.30 & -1.15 \\
\hline & & & Mean & Min & $\operatorname{Max}$ & & & \\
\hline A & $18(7)$ & 0 & -3.86 & -5.34 & -2.28 & $\ldots$ & $\ldots$ & $\ldots$ \\
\hline $\mathrm{WD}^{\mathrm{b}}$ & $8(7)$ & 1 & -0.04 & -0.86 & $\begin{array}{l}2.20 \\
+0.65\end{array}$ & $\ldots$ & $\ldots$ & $\ldots$ \\
\hline $\mathrm{CV}$ & $4(4)$ & 0 & $-0.73^{\mathrm{c}}$ & $-2.04^{\mathrm{c}}$ & $-0.12^{\mathrm{c}}$ & $\ldots$ & $\ldots$ & $\ldots$ \\
\hline XRB & $1(1)$ & 0 & +1.18 & $\ldots$ & $\ldots$ & $\ldots$ & $\ldots$ & $\ldots$ \\
\hline
\end{tabular}

Notes. The number in parentheses in the second column is the number of known X-ray emitters. Empirical $2 \sigma \log \left(f_{X} / f_{V}\right)$ ranges and means are given for reliable samples of F, G, K, and M stars. The full flux ratio ranges are given for the A stars, WDs, and CVs.

${ }^{\text {a }}$ Six are identified based on their $\left(f_{X} / f_{V}\right)$; three without a cataloged $V$ are identified based on their $\left(f_{X} / f_{J}\right)$.

b Only six WDs have cataloged $V$ magnitudes.

${ }^{\mathrm{c}}$ These are $\log \left(f_{X} / f_{B}\right)$ values.

sources. The Maccacaro et al. (1988) Einstein limits are also included in each panel. The flux ratio distributions and ranges for the SIMBAD stars are consistent with those found in these earlier studies. This suggests that, as expected, the vast majority of these stars are RASS source counterparts. Furthermore, the flux ratio ranges calculated from our own distributions for these stellar types give us limits slightly more generous than the Einstein values (which are known to be quite conservative; Stocke et al. 1991), but generally more conservative than those of Krautter et al. (1999).

Table 1 summarizes the properties of our sample of SIMBAD stars. We list the total number of stars of each spectral type in the initial sample, the number of known X-ray emitters among them, and the number of new X-ray source counterparts we identify based on the combination of positional proximity and appropriate X-ray-to-optical flux ratios. For the F, G, K, and $\mathrm{M}$ stars, we include the empirically derived $\log \left(f_{X} / f_{V}\right)$ means and $2 \sigma$ ranges. For the A stars, WDs, and CVs, which are less numerous, we list the full range of flux ratios and their means. For the CVs, we give $\log \left(f_{X} / f_{B}\right)$ (see Zickgraf et al. 2003).

Below we touch on the properties of the CVs and WDs in this sample, and discuss our use of the F, G, K, and M stars to construct empirical $\log \left(f_{X} / f_{J}\right)$ ranges. ${ }^{17}$

\footnotetext{
17 The A stars are discussed in Appendix A.
} 
Table 2

The CVs and WDs Among the RASS/SIMBAD Matches

\begin{tabular}{|c|c|c|c|c|c|c|}
\hline $1 \mathrm{RXS} \mathrm{J}$ & Counts s ${ }^{-1}$ & Counterpart & $\begin{array}{c}\text { Offset } \\
\left({ }^{\prime \prime}\right)\end{array}$ & $\begin{array}{c}B \\
(\mathrm{mag})\end{array}$ & $\begin{array}{c}V \\
(\mathrm{mag})\end{array}$ & Type \\
\hline $015543.3+002817$ & $0.042 \pm 0.012$ & $\mathrm{~V} * \mathrm{FL}$ Cet & 9.9 & 16.60 & 15.50 & $\mathrm{P}$ \\
\hline $083821.6+483800$ & $0.565 \pm 0.042$ & $\mathrm{~V} * \mathrm{EI} \mathrm{UMa}$ & 4.2 & 14.90 & $\ldots$ & $\mathrm{DN}$ \\
\hline $085343.5+574846$ & $0.414 \pm 0.035$ & $\mathrm{~V} * \mathrm{BZ} \mathrm{UMa}$ & 8.0 & 15.30 & $\ldots$ & $\mathrm{DN}$ \\
\hline $113826.8+032210$ & $0.659 \pm 0.061$ & $\mathrm{~V} * \mathrm{~T}$ Leo & 2.9 & 10.00 & 10.00 & $\mathrm{DN}$ \\
\hline $020801.8+133625$ & $0.054 \pm 0.016$ & WD $0205+133$ & 24.7 & 13.78 & $\ldots$ & $\mathrm{DA}+\mathrm{dM} 1$ \\
\hline $034850.1-005823$ & $1.965 \pm 0.158$ & WD $0346-011$ & 9.2 & 13.82 & 13.98 & DA \\
\hline $084104.2+032118$ & $1.207 \pm 0.065$ & WD $0838+035$ & 5.1 & $\ldots$ & 14.48 & DA \\
\hline $112814.4-024950$ & $0.051 \pm 0.015$ & & 33.1 & 15.32 & $\ldots$ & DA \\
\hline $152146.6+522215$ & $0.168 \pm 0.016$ & WD $1520+525$ & 11.6 & 15.56 & 16.60 & DO \\
\hline $165008.0+370130$ & $0.115 \pm 0.019$ & WD $1648+371$ & 20.3 & $\ldots$ & 16.10 & DA \\
\hline $165020.4+403723$ & $0.094 \pm 0.016$ & WD $1648+407$ & 3.9 & $\ldots$ & 14.50 & DA \\
\hline $172642.8+583726$ & $1.247 \pm 0.028$ & WD $1725+586$ & 7.7 & 15.36 & 15.45 & DA \\
\hline
\end{tabular}

Notes. "P" indicates that CV is a polar and "DN" that it is a dwarf nova.

\subsubsection{The CVs and WDs}

Table 2 lists the matched RASS/SIMBAD CVs and WDs. All of the CVs are known X-ray emitters; only EI UMa does not have an SDSS spectrum. Szkody et al. (2002) and Schmidt et al. (2005b) identified FL Cet as a polar, where accretion from (typically) a late-type star is directly onto a WD's magnetic poles. The three other CVs are DNe.

Six of the eight WDs in Table 2 are listed as X-ray sources by Fleming et al. (1996) while another, WD $1520+525$, was proposed as a soft X-ray source by Chu et al. (2004). Seven of these WDs are DAs, stars with pure hydrogen atmospheres that are the most commonly observed WD type. WD $1520+525$ is the only non-DA; its spectrum includes strong He II features, making it a DO WD. Wachter et al. (2003) identified WD $0205+133$ as belonging to a binary system on the basis of its infrared excess and Farihi et al. (2006) resolved its M-star companion; this WD system is the only one not to be previously proposed as an X-ray source.

Overall, the X-ray sources near the WDs have very soft X-ray spectra, as measured by their hardness ratios. For these sources, $\mathrm{HR} 1 \approx-1$, which is typical for X-ray-emitting WDs (e.g., Fleming et al. 1996; Zickgraf et al. 1997), and confirms that their counterparts are indeed likely to be these WDs. Only one of these objects has an SDSS spectrum: WD $1725+586$.

For a broader discussion of RASS detections of WDs and CVs, see Section 6.

\subsubsection{Using the Known Stars to Construct Empirical $\log \left(f_{X} / f_{J}\right)$ Ranges}

While typical flux ratio ranges can be derived using log $\left(f_{X} / f_{V}\right)$ calculations for our sample of previously cataloged stars, we cannot use this flux ratio to evaluate the likelihood that fainter, previously uncataloged SDSS stars are X-ray emitters, as they generally lack SIMBAD $V$ magnitudes. ${ }^{18}$ While $g$ or $r$ would be a natural substitute for $V$, because many of these stars are saturated, they have unreliable SDSS photometry. Instead, we use 2MASS $J$ magnitudes; all of the stars in our sample have a 2MASS counterpart.

To calculate flux ratios, we adapt the Maccacaro et al. (1988) formula to reflect the definition of the 2MASS J-band flux (e.g.,

\footnotetext{
18 A handful do: a few SIMBAD entries give a magnitude but no spectral class, for example. These stars are included in the APO spectroscopic sample discussed in Section 4.2.
}

Cohen et al. 2003): $\log \left(f_{X} / f_{J}\right)=\log \left(f_{X}\right)+0.4 J+6.30$. The SIMBAD stars included in our $\left(f_{X} / f_{J}\right)$ calculations are either previously known X-ray emitters or newly identified RASS counterparts, as defined in Section 4.1.1; in addition, we require that the $J$-band errors $\leqslant 0.11 \mathrm{mag}$ (for a discussion of the 2MASS flags, see Cutri et al. 2003).

As mentioned above, a small number of these stars have both a cataloged spectral type and one derived from an APO spectrum. In 24 cases, using either type has no effect on the $\left(f_{X} / f_{J}\right)$ distributions; in 13 cases it does. Eleven of these stars were classified as "F/G" or "K" by Zickgraf et al. (2003); these spectra are lower resolution than ours, and we therefore use our APO-derived types instead. The other stars are V* V842 Her and StKM 1-1262, discussed above; we used the SIMBAD types for these two.

We then have $125 \mathrm{~F}, 94 \mathrm{G}, 76 \mathrm{~K}$, and $42 \mathrm{M}$ stars with which to construct empirical $\log \left(f_{X} / f_{J}\right)$ ranges. The distributions are shown in Figure 5. The derived flux ratio $2 \sigma$ ranges and means are listed in Table 1 alongside the earlier $\log \left(f_{X} / f_{V}\right)$ values. Based on their $\log \left(f_{X} / f_{J}\right)$ values, we are able to add three of the SIMBAD M stars lacking a cataloged $V$ and previously unassociated with RASS sources to our list of new stellar X-ray emitters. In total, we have identified $104 \mathrm{~F}, 70 \mathrm{G}, 47 \mathrm{~K}$, and $9 \mathrm{M}$ stars cataloged in SIMBAD but previously unassociated with an $\mathrm{X}$-ray source as RASS source counterparts. These are included in our final catalog; see Section 5.

\subsection{The APO Spectroscopic Sample}

Our search for cataloged candidate counterparts identified $\sim 1400$ RASS sources with no obvious counterparts in either SIMBAD or NED. ${ }^{19}$ The objects $\leqslant 30^{\prime \prime}$ from a RASS source made up the list of primary targets for our spectroscopic campaign. We used the Dual Imaging Spectrograph (DIS) on the APO $3.5 \mathrm{~m}$ telescope to obtain spectra for $\sim 95 \%$ of these objects, and for $>100$ objects with separations $>30^{\prime \prime}$, over the course of 50 half nights from 2003 September to 2007 July. In our standard setup, the DIS "low" blue grating is centered on $4500 \AA$ (with a dispersion of $2.4 \AA$ pixel $^{-1}$ ); its "medium" red grating is centered on $7600 \AA\left(2.3 \AA\right.$ pixel $\left.^{-1}\right)$; we use a 1".5 (3 pixels) slit. This results in wavelength coverage from 3800 to $8700 \AA$ with a resolution of $\sim 700$ in the blue and

\footnotetext{
19 Some sources with matches were included, e.g., matches to Tycho stars
} lacking a published spectral type. 

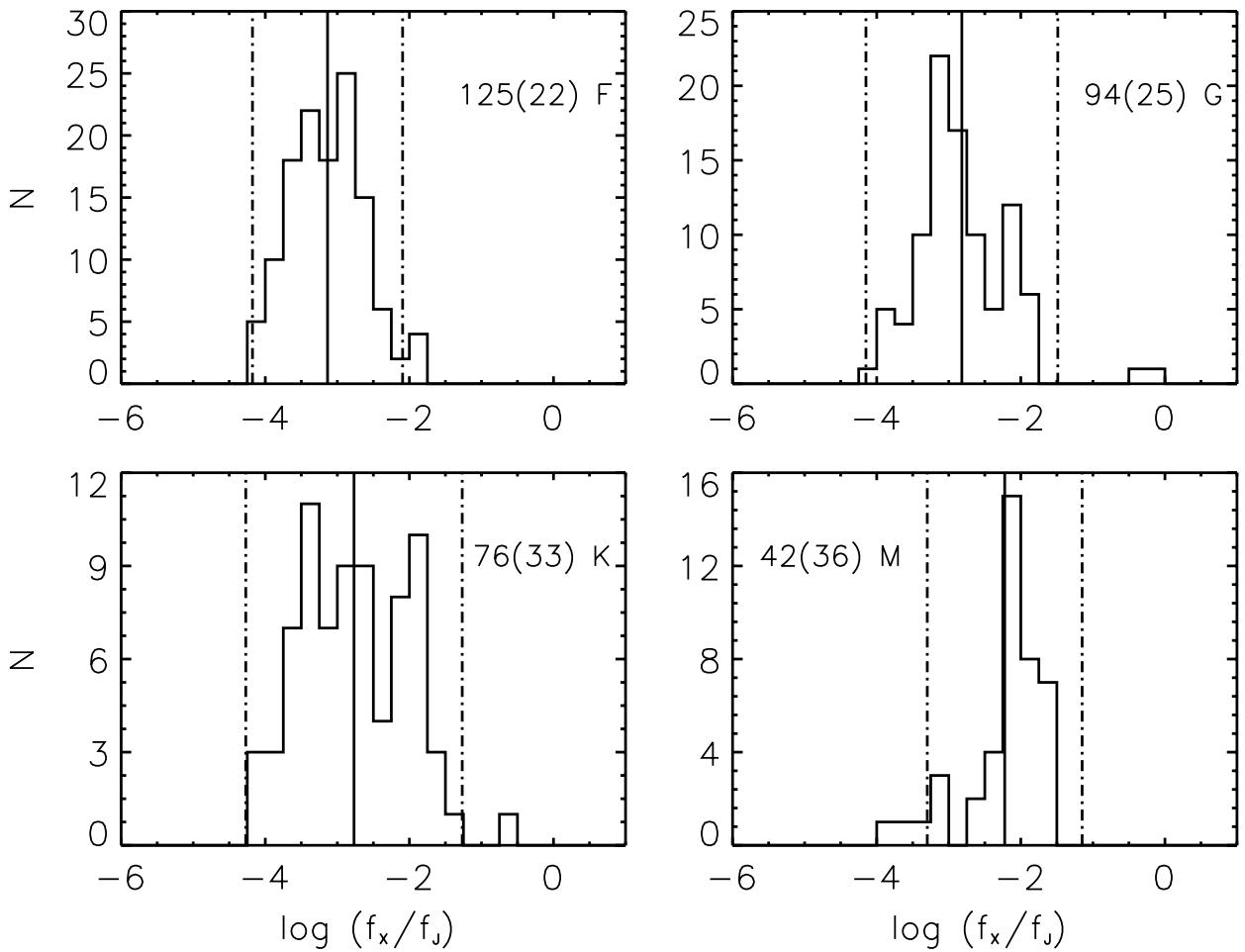

Figure 5. $\log \left(f_{X} / f_{J}\right)$ ratios for the RASS/SIMBAD F, G, K, and M stars. As in Figure 4, the solid vertical lines correspond to the mean $\left(f_{X} / f_{J}\right)$ and the dot-dashed vertical lines bracket the $2 \sigma\left(f_{X} / f_{J}\right)$ range for each stellar type. The number of RASS/SIMBAD stars used to calculate these $\left(f_{X} / f_{J}\right)$ ranges is indicated in each panel; the number in parentheses is the subset of previously known X-ray emitters.

$\sim 1000$ in the red. Almost all of our observing was done in bright time, and our integrations were typically $\lesssim 5$ minutes. The data were flux calibrated with standards taken each night. The spectra were reduced with a script written in PyRAF, the Python-based command language for the Image Reduction and Analysis Facility (IRAF). ${ }^{20}$ All spectra were trimmed, overscan, and bias corrected, cleaned of cosmic rays, flat fielded, extracted, dispersion corrected, and flux calibrated using standard IRAF tasks.

In most cases, a single observation was sufficient to obtain a spectral type for the candidate counterpart; we thus have 649 error circles with a unique spectrum. In 107 error circles we took at least two spectra of the same target, generally because the target was relatively faint $(g \gtrsim 16)$ or the weather uncooperative, and the first spectrum was of insufficient quality to allow for confident typing. In an additional 98 RASS error circles we took spectra for more than one target, usually because of the presence of an apparent companion to our target. The total number of RASS sources considered is nearly 900 if we include the 41 SIMBAD stars with cataloged spectral types for which we obtained APO spectra.

To obtain spectral types for our targets, we used the Hammer (Covey et al. 2007). The Hammer automatically predicts the Morgan-Keenan (for stars earlier than M) or Kirkpatrick (for later stars) spectral type on the basis of a fit to a set of 30 spectral indices. ${ }^{21}$ The Hammer also allows the user to interactively modify the assigned spectral type. Every spectrum was therefore checked by eye before a final type was assigned.

\footnotetext{
${ }^{20}$ PyRAF is a product of the Space Telescope Science Institute, which is operated by AURA for NASA. IRAF is distributed by the National Optical Astronomy Observatories, which are operated by the Association of Universities for Research in Astronomy, Inc., under cooperative agreement with the National Science Foundation.

21 The Hammer is written in IDL and is available from

http://www.cfa.harvard.edu/ kcovey/.
}

As noted above, in 107 cases we obtained at least two spectra of a candidate RASS counterpart, generally because our first spectrum was fairly noisy. Nevertheless, $80 \%$ of the time the stellar types assigned to a given star agree to within two subclasses, which we take to be our uncertainty. For those cases where the difference is larger, we assigned the spectral type obtained from fitting the highest signal-to-noise spectrum for that particular star.

In Figure 6, we present the positional offset and $\sigma$ (as defined earlier) distributions for stars in 751 of the 756 RASS error circles where we selected only one target. For these calculations, we updated the positional (and photometric) data for the SDSS stars to the more recent Data Release 6 (DR6; AdelmanMcCarthy et al. 2008) values. Of the five objects not included, one is a QSO, two are stars whose spectra are too poor for confident typing, and two others lack data in DR6 because they fall on the survey edges.

646 of the 751 stars have separations $\leqslant 30^{\prime \prime}$; to these we add two stars with separations $>30^{\prime \prime}$ previously identified as RASS counterparts by Zickgraf et al. (2003) but lacking a spectral type. Of these 648 stars, 645 are F, G, K, or M stars (two others were typed as A stars ${ }^{22}$ and one is a CV). If we apply the same $J$-band quality cut as to our earlier sample $\left(J_{\text {err }} \leqslant 0.11\right.$ $\mathrm{mag}$ ), and remove objects for which the RASS flag is not 0 , we are left with a sample of 616 stars: 70 F, 245 G, $166 \mathrm{~K}$, and $132 \mathrm{M}$.

The $\log \left(f_{X} / f_{J}\right)$ distributions for these $\mathrm{F}, \mathrm{G}, \mathrm{K}$, and $\mathrm{M}$ stars are plotted in Figure 7. We also include the flux ratio means and $2 \sigma$ ranges derived from the RASS/SIMBAD sample (see Table 1). We identify as spectroscopically confirmed counterparts to RASS X-ray sources objects with $\left(f_{X} / f_{J}\right)$ within $2 \sigma$ of the mean $\left(f_{X} / f_{J}\right)$ value of optically bright X-ray emitters in the same spectral-type range. These plots show clearly that the

\footnotetext{
22 Discussed in Appendix A.
} 

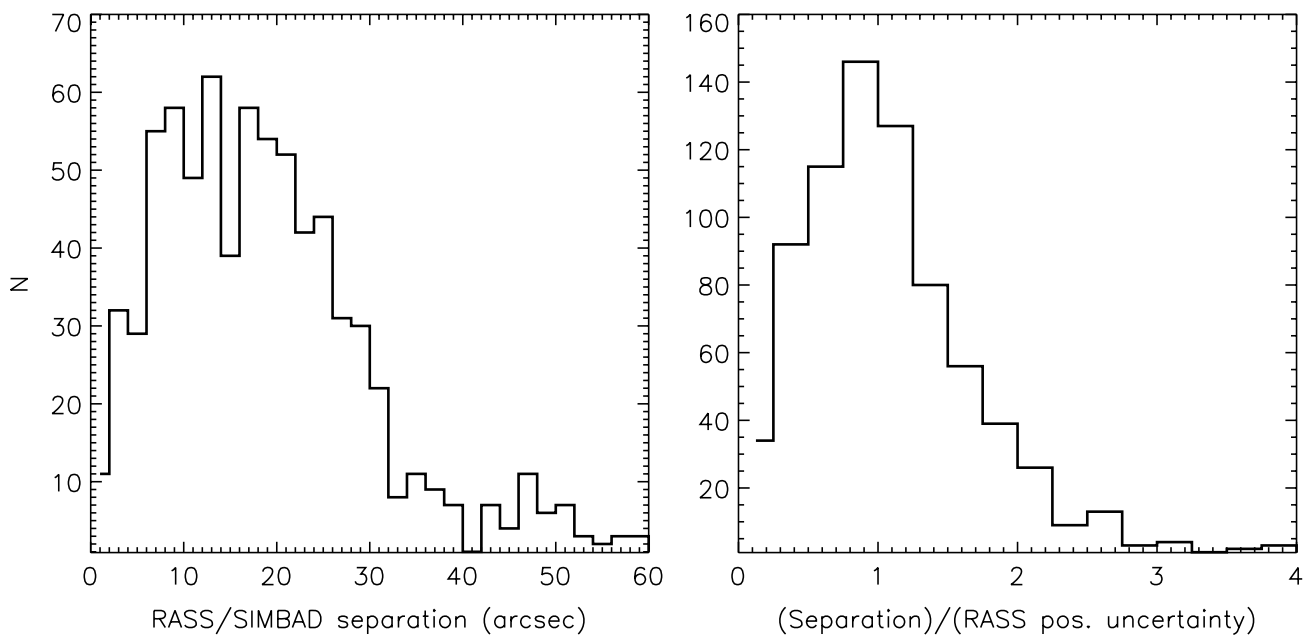

Figure 6. Left panel: distribution of positional offsets between the RASS sources and 751 candidate SDSS stellar counterparts with APO spectra. Right panel: the same distribution, expressed in terms of the cataloged X-ray positional uncertainty.
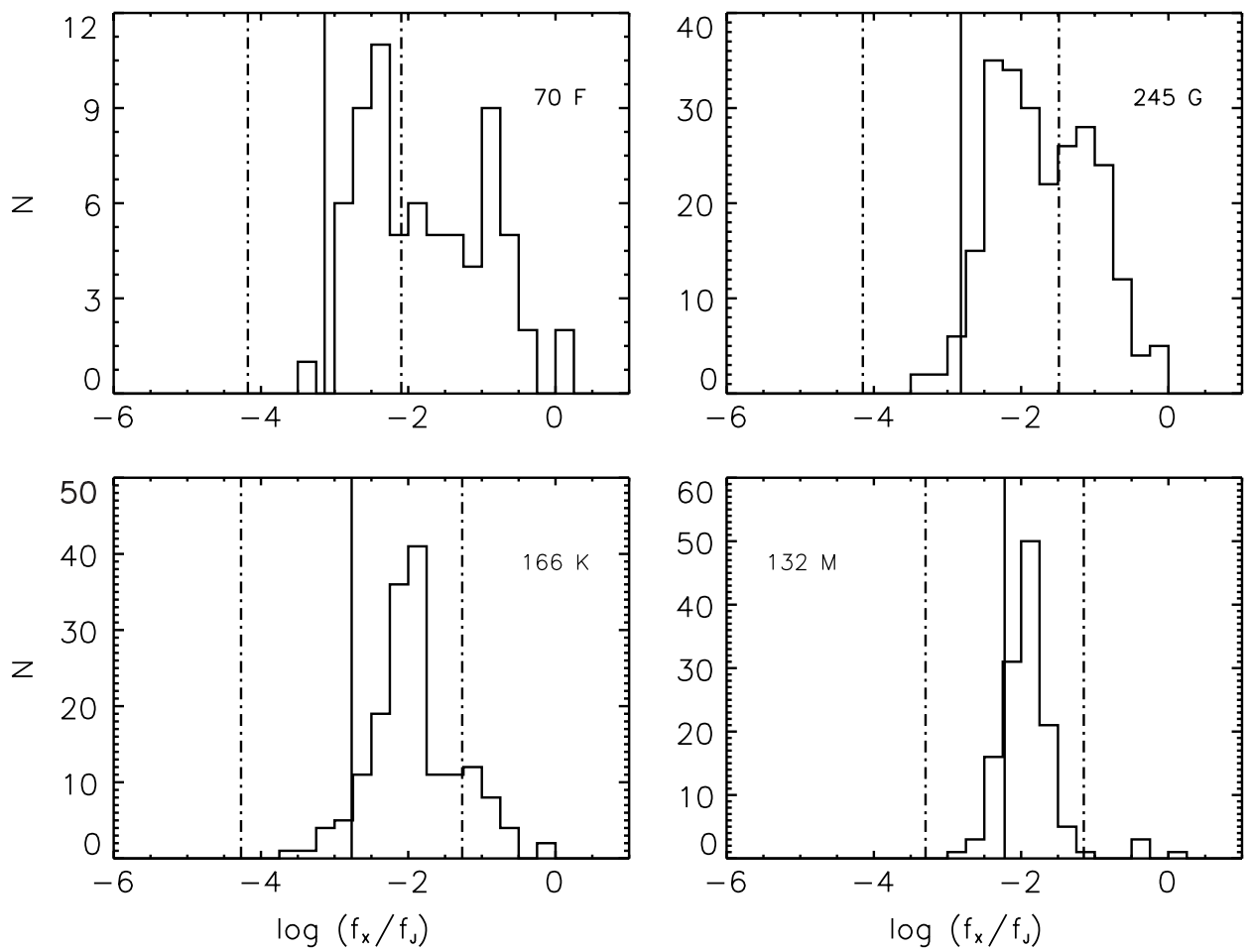

Figure 7. $\log \left(f_{X} / f_{J}\right)$ ratios for the candidate SDSS counterparts with APO spectroscopy. The solid vertical lines correspond to the mean $\left(f_{X} / f_{J}\right)$ and the dot-dashed vertical lines bracket the $2 \sigma\left(f_{X} / f_{J}\right)$ range for each stellar type as derived from the RASS/SIMBAD sample. The number of SDSS/APO stars used to calculate these $\left(f_{X} / f_{J}\right)$ ranges is indicated in each panel.

probability that a spectroscopic target is a RASS counterpart increases as we move to later spectral types. Only 31 of the 70 spectroscopically identified $\mathrm{F}$ stars fall within the $2 \sigma \log$ $\left(f_{X} / f_{J}\right)$ range derived from the RASS/SIMBAD F stars $(44 \%)$; for the $\mathrm{G}$ stars, the fraction is 149 of $245(61 \%)$. For $\mathrm{K}$ stars and $\mathrm{M}$ stars the fraction is much higher: 139 of $166 \mathrm{~K}$ stars $(84 \%)$ and 127 of $132 \mathrm{M}$ stars $(96 \%)$ have $\log \left(f_{X} / f_{J}\right)$ within their respective $2 \sigma$ ranges.

The X-ray, optical, and infrared properties of the 443 new $F, G, K$, and $M X$-ray emitters identified here are included in our final catalog, ${ }^{23}$ described in Section 5,

\footnotetext{
${ }^{23}$ Note that this number reflects our removal of three stars (one G, one $\mathrm{K}$, and one $\mathrm{M}$ ) observed at APO from our final catalog based on our comparison to the Flesch \& Hardcastle (2004) catalog. See Section 5.2.
}

along with the 230 RASS/SIMBAD F, G, K, and M stars identified above. Below we discuss the error circles for which we obtained multiple APO spectra, our candidate flare stars, and a new CV discovered in this spectroscopic campaign.

\subsubsection{The Error Circles with Multiple Spectra}

For the error circles in which we have APO spectra for several targets, we calculated the positional separation and $\log \left(f_{X} / f_{J}\right)$ ratio and obtained a spectral type for each. The best candidate counterpart in each error circle was then identified on the basis of its proximity (only stars $\leqslant 30^{\prime \prime}$ from a RASS source whose $\mathrm{X}$-ray flag is 0 were considered) and the appropriateness of its $\left(f_{X} / f_{J}\right)$ given its spectral type. In 34 error circles, we identified 
Table 3

RASS Sources with Multiple Candidate Counterparts

\begin{tabular}{|c|c|c|c|c|c|c|c|c|}
\hline $1 \mathrm{RXS} \mathrm{J}$ & Counts s $^{-1}$ & SDSS J & $\begin{array}{c}\text { Offset } \\
\left({ }^{\prime \prime}\right)\end{array}$ & $\begin{array}{c}g \\
(\mathrm{mag}) \\
\end{array}$ & $\begin{array}{c}J \\
(\mathrm{mag}) \\
\end{array}$ & $\begin{array}{c}\log \\
\left(f_{X} / f_{J}\right)\end{array}$ & $\begin{array}{l}\text { APO } \\
\text { type }\end{array}$ & $\begin{array}{c}\text { SIMBAD } \\
\text { data }\end{array}$ \\
\hline \multirow[t]{2}{*}{$002203.7-101628$} & $0.135 \pm 0.024$ & $002204.47-101619.1$ & 14.5 & $12.98 \pm 0.01$ & $9.51 \pm 0.02$ & -1.77 & K7e & $\ldots$ \\
\hline & & $002204.20-101613.2$ & 16.5 & $14.55 \pm 0.05$ & $10.00 \pm 0.02$ & -1.57 & K5 & $\ldots$ \\
\hline \multirow[t]{2}{*}{$083203.9+530354$} & $0.020 \pm 0.010$ & $083203.74+530359.6$ & 5.8 & $12.33 \pm 0.02$ & $10.08 \pm 0.02$ & -2.37 & $\mathrm{~K} 1$ & $\ldots$ \\
\hline & & $083202.66+530400.0$ & 12.7 & $13.48 \pm 0.13$ & $10.27 \pm 0.02$ & -2.30 & G0 & $\ldots$ \\
\hline \multirow{2}{*}{$092455.1+575649$} & $0.130 \pm 0.023$ & $092456.44+575652.8$ & 11.2 & $14.09 \pm 0.02$ & $9.83 \pm 0.02$ & -1.66 & M3 & $\ldots$ \\
\hline & & $092455.33+575700.0$ & 10.7 & $14.62 \pm 0.08$ & $8.66 \pm 0.02$ & -2.12 & M2 & [ZEH2003] RX J0924.9+5756 1; * \\
\hline \multirow[t]{2}{*}{$110830.8+011742$} & $0.073 \pm 0.015$ & $110830.87+011741.6$ & 1.4 & $11.92 \pm 0.00$ & $10.28 \pm 0.03$ & -1.73 & G7 & $\ldots$ \\
\hline & & $110830.87+011741.6$ & 1.4 & $11.92 \pm 0.00$ & $10.35 \pm 0.07$ & -1.70 & $\mathrm{G} 2$ & RX J1108.5 + 0117 \\
\hline \multirow[t]{2}{*}{$114930.0-032623$} & $0.052 \pm 0.016$ & $114930.34-032632.4$ & 10.8 & $11.37 \pm 0.00$ & $9.53 \pm 0.03$ & -2.17 & G0 & $\ldots$ \\
\hline & & $114930.34-032632.4$ & 10.8 & $11.37 \pm 0.00$ & $9.53 \pm 0.03$ & -2.17 & G6 & $\ldots$ \\
\hline \multirow[t]{2}{*}{$123247.7-010419$} & $0.024 \pm 0.010$ & $123247.68-010419.8$ & 0.4 & $14.38 \pm 0.20$ & $10.11 \pm 0.03$ & -2.28 & G6 & $\ldots$ \\
\hline & & $123247.28-010423.7$ & 7.5 & $15.37 \pm 0.15$ & $10.69 \pm 0.03$ & -2.05 & G8 & $\ldots$ \\
\hline \multirow[t]{2}{*}{$132339.1+641635$} & $0.021 \pm 0.009$ & $132338.98+641630.2$ & 4.8 & $15.10 \pm 0.07$ & $11.4 \pm 0.03$ & -1.81 & M0 & $\ldots$ \\
\hline & & $132339.64+641630.4$ & 5.8 & $13.90 \pm 0.03$ & $10.67 \pm 0.05$ & -2.11 & M1 & $\cdots$ \\
\hline \multirow[t]{2}{*}{$135902.2+591754$} & $0.067 \pm 0.013$ & $135902.75+591744.4$ & 10.4 & $12.32 \pm 0.00$ & $9.42 \pm 0.03$ & -2.11 & K5 & GPM $209.761906+59.296354 ; 11.30 ;-2.11 ; *$ \\
\hline & & $135903.24+591747.4$ & 10.3 & $16.21 \pm 0.07$ & $11.52 \pm 0.03$ & -1.27 & M3 & $\ldots$ \\
\hline \multirow[t]{2}{*}{$140148.9+001008$} & $0.024 \pm 0.011$ & $140148.48+001024.7$ & 17.9 & $14.35 \pm 0.11$ & $11.03 \pm 0.03$ & -1.90 & K4 & $\ldots$ \\
\hline & & $140148.69+001025.5$ & 17.8 & $14.42 \pm 0.02$ & $10.75 \pm 0.06$ & -2.02 & M0 & $\ldots$ \\
\hline \multirow[t]{2}{*}{$150656.8+011533$} & $0.025 \pm 0.010$ & $150656.87+011532.7$ & 1.4 & $15.37 \pm 0.02$ & $10.84 \pm 0.05$ & -1.97 & M3 & $\ldots$ \\
\hline & & $150656.90+011527.0$ & 6.6 & $16.65 \pm 0.12$ & $11.31 \pm 0.03$ & -1.78 & M3 & $\cdots$ \\
\hline \multirow[t]{2}{*}{$153826.3+523407$} & $0.010 \pm 0.004$ & $153826.02+523414.0$ & 7.5 & $17.88 \pm 14.96$ & $9.11 \pm 0.04$ & -3.06 & $\mathrm{~K} 3$ & $\mathrm{BD}+53$ 1797B; $10.70 ;-3.06 ; * \mathrm{i} *$ \\
\hline & & $153826.38+523418.2$ & 11.3 & $14.38 \pm 0.16$ & $8.88 \pm 0.03$ & -3.15 & $\mathrm{~K} 3$ & HD $234250 ; 9.50 ;-3.15 ; \mathrm{K} 2 ; * \mathrm{i} *$ \\
\hline \multirow[t]{2}{*}{$164129.8+400617$} & $0.022 \pm 0.009$ & $164129.54+400559.5$ & 17.7 & $20.02 \pm 13.33$ & $8.92 \pm 0.04$ & -2.79 & G8 & $\mathrm{BD}+403051^{\mathrm{a}} ; *$ \\
\hline & & $164129.47+400602.9$ & 14.5 & $10.15 \pm 0.00$ & $8.92 \pm 0.04$ & -2.79 & G5 & $\ldots$ \\
\hline \multirow[t]{2}{*}{$171017.5+632135$} & $0.052 \pm 0.006$ & $171017.05+632141.1$ & 6.8 & $13.23 \pm 0.07$ & $9.77 \pm 0.03$ & -2.08 & $\mathrm{~K} 4$ & $2 \mathrm{E} 1709.9+6325$ \\
\hline & & $171016.46+632133.9$ & 7.1 & $19.96 \pm 6.98$ & $10.05 \pm 0.02$ & -1.96 & M0 & $2 \mathrm{E} 1709.9+6325$ \\
\hline \multirow[t]{2}{*}{$235942.7-011325$} & $0.029 \pm 0.011$ & $235943.68-011322.7$ & 15.0 & $15.85 \pm 0.11$ & $9.94 \pm 0.05$ & -2.26 & K7 & $\ldots$ \\
\hline & & $235943.80-011325.6$ & 16.6 & $14.18 \pm 0.03$ & $10.47 \pm 0.03$ & -2.05 & K3 & $\ldots$ \\
\hline \multirow[t]{2}{*}{$001619.1-010459$} & $0.020 \pm 0.009$ & $001620.75-010504.6$ & 25.5 & $14.85 \pm 0.03$ & $12.57 \pm 0.03$ & -1.37 & $\mathrm{~K} 3 \mathrm{e}$ & GSC 04664-01105; * \\
\hline & & $001620.16-010506.5$ & 17.6 & $15.51 \pm 0.03$ & $12.27 \pm 0.02$ & -1.49 & K7 & $\ldots$ \\
\hline \multirow[t]{2}{*}{$015119.8+132459$} & $0.172 \pm 0.023$ & $015119.97+132512.4$ & 13.2 & $11.86 \pm 0.00$ & $9.08 \pm 0.02$ & -1.83 & G9 & [ZEH2003] RX J0151.3+ 13242 \\
\hline & & $015119.97+132452.2$ & 7.7 & $14.85 \pm 0.08$ & $8.56 \pm 0.03$ & -2.04 & M3 & $\ldots$ \\
\hline \multirow[t]{2}{*}{$020419.0+131851$} & $0.053 \pm 0.016$ & $020419.12+131856.0$ & 5.4 & $18.37 \pm 92.46$ & $9.98 \pm 0.02$ & -1.99 & M3 & [ZEH2003] RX J0204.3 + 13182 \\
\hline & & $020418.31+131853.3$ & 10.3 & $16.00 \pm 0.01$ & $11.33 \pm 0.02$ & -1.45 & K0 & $\ldots$ \\
\hline \multirow[t]{2}{*}{$085921.0+503256$} & $0.022 \pm 0.009$ & $085922.99+503307.6$ & 22.3 & $12.63 \pm 0.00$ & $10.85 \pm 0.03$ & -2.03 & G8 & $\cdots$ \\
\hline & & $085922.79+503311.4$ & 23.1 & $16.61 \pm 0.24$ & $12.92 \pm 0.05$ & -1.20 & M0 & $\ldots$ \\
\hline $091140.6+493319$ & $0.018 \pm 0.008$ & $091139.52+493326.7$ & 12.7 & $17.63 \pm 0.02$ & $12.77 \pm 0.02$ & -1.34 & M4 & $\ldots$ \\
\hline & & $091140.92+493325.8$ & 7.1 & $15.36 \pm 0.02$ & $11.67 \pm 0.02$ & -1.78 & M1 & $\ldots$ \\
\hline $145626.1+560341$ & $0.019 \pm 0.007$ & $145628.50+560321.9$ & 28.0 & $10.96 \pm 0.00$ & $9.60 \pm 0.02$ & -2.57 & G0 & TYC $3864-410-1 ; 10.63 ;-2.57 ; *$ \\
\hline & & $145626.46+560324.6$ & 17.2 & $13.55 \pm 0.01$ & $10.69 \pm 0.02$ & -2.13 & K5 & $\ldots$ \\
\hline $150521.8+595810$ & $0.010 \pm 0.004$ & $150523.09+595755.3$ & 18.0 & $15.69 \pm 0.18$ & $10.01 \pm 0.02$ & -2.70 & G6 & $\ldots$ \\
\hline & & $150520.38+595755.3$ & 18.5 & $11.25 \pm 0.00$ & $9.83 \pm 0.02$ & -2.78 & G6 & $\ldots$ \\
\hline $152350.6+533009$ & $0.018 \pm 0.007$ & $152348.89+532959.2$ & 18.3 & $13.72 \pm 0.01$ & $9.33 \pm 0.02$ & -2.72 & M3 & NLTT 40162; PM* \\
\hline & & $152351.61+532942.0$ & 28.9 & $18.91 \pm 0.02$ & $\ldots$ & $+0.19^{\mathrm{b}}$ & QSO & $\ldots$ \\
\hline $160207.9+534105$ & $0.013 \pm 0.006$ & $160206.84+534039.9$ & 27.2 & $12.25 \pm 0.00$ & $7.71 \pm 0.02$ & -3.49 & G9 & TYC $3877-1522-1 ; 9.61 ;-3.49 ; *$ \\
\hline & & $160208.39+534122.5$ & 17.6 & $13.84 \pm 0.02$ & $11.47 \pm 0.02$ & -1.99 & K1 & $\cdots$ \\
\hline $163845.2+392324$ & $0.022 \pm 0.007$ & $163845.73+392320.4$ & 7.4 & $12.41 \pm 0.00$ & $10.45 \pm 0.02$ & -2.18 & $\mathrm{~K} 2$ & $\cdots$ \\
\hline & & $163844.64+392308.5$ & 17.2 & $15.36 \pm 0.01$ & $12.27 \pm 0.02$ & -1.46 & K7 & $\ldots$ \\
\hline $171702.0+561220$ & $0.047 \pm 0.007$ & $171702.55+561235.6$ & 15.8 & $13.40 \pm 0.02$ & $9.52 \pm 0.02$ & -2.22 & G6 & $\ldots$ \\
\hline & & $171702.12+561219.2$ & 1.6 & $13.99 \pm 0.05$ & $8.62 \pm 0.04$ & -2.58 & $\mathrm{~K} 2$ & TYC 3891-410-1; $10.24 ;-2.58 ; *$ \\
\hline $172713.4+593324$ & $0.013 \pm 0.004$ & $172715.09+593315.1$ & 16.0 & $15.42 \pm 0.02$ & $11.3 \pm 0.02$ & -2.08 & G7 & $\ldots$ \\
\hline & & $172714.58+593338.5$ & 16.7 & $10.63 \pm 0.00$ & $8.97 \pm 0.02$ & -3.02 & G8 & TYC 3900-417-1; 10.30; -3.02; * \\
\hline
\end{tabular}

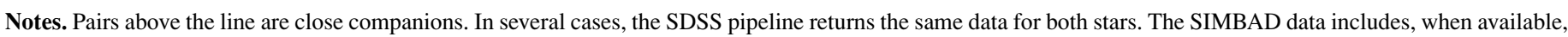

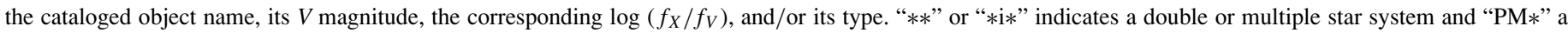
high proper-motion star.

a The given SIMBAD position is offset by $37^{\prime \prime}$, but this appears to be the SDSS star.

${ }^{\mathrm{b}} \log \left(f_{X} / f_{V}\right)$. There is no 2 MASS detection of this QSO.

a single stellar counterpart ( $2 \mathrm{~F}, 11 \mathrm{G}, 8 \mathrm{~K}$, and $13 \mathrm{M}$ stars); these are included in our final catalog.

In another 26 error circles, two stars met our criteria; in 14 of these cases our target has an apparent close companion (separations of $\sim 5^{\prime \prime}$ or less), making it difficult to avoid photometric uncertainties in the SDSS and 2MASS catalogs and sometimes to obtain clean spectra with our 1".5 slit. We list these error circles separately in Table 3. Higher-resolution X-ray 

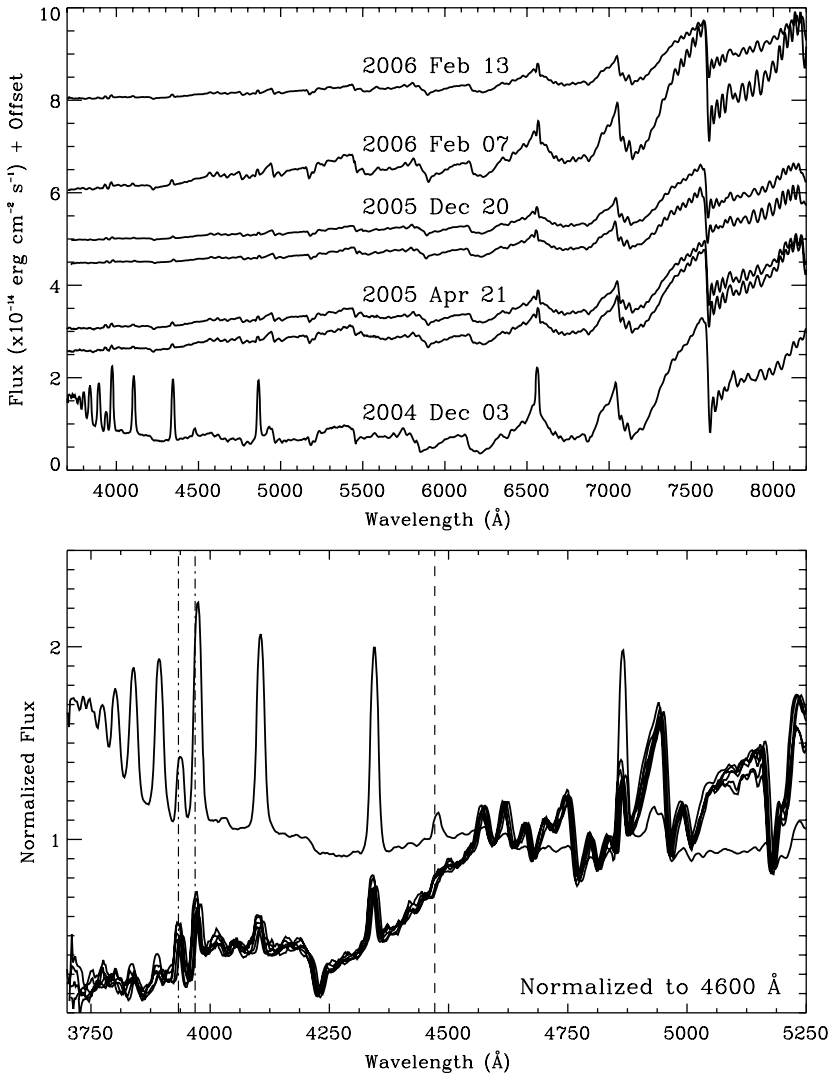

Figure 8. APO spectra of the proposed stellar counterpart to 1 RXS J080826.7+434745. Top: the spectra are artificially offset from each other in the flux; two spectra were obtained on 2005 April 21 and December 20. Bottom: the blue continuum and strong Balmer emission visible in the 2004 December 3 spectrum are even more apparent when all of the spectra are normalized to their flux value at $4600 \AA$. The dot-dashed lines indicate the positions of the $\mathrm{Ca} \mathrm{H} \&$ $\mathrm{K}$ lines (at this resolution, $\mathrm{Ca} \mathrm{H}$ is blended with $\mathrm{H} \epsilon$ ), while the dashed line is He I (4471 ̊).

observations are required to determine which of the possible counterparts is in fact the X-ray source; it is also possible, of course, that both stars are X-ray emitters.

\subsubsection{The Candidate Flare Stars}

Many of the stars for which we obtained multiple spectra were $M$ stars with what appeared to be stronger-than-average emission lines. Figure 8 shows the spectra for the target with the largest number of observations, the proposed counterpart to 1RXS J080826.7+434745. The initial spectrum suggested that we caught this M4 star flaring, and we returned to it regularly over the course of the next two years. All of the spectra include the Balmer and $\mathrm{Ca}$ II $\mathrm{H}$ and $\mathrm{K}$ emission lines typical of $\mathrm{M}$ star spectra. However, these features are much stronger in the initial spectrum, as can be clearly seen in the bottom panel of Figure 8, where the spectra have been normalized to the value of their flux at $4600 \AA$. In addition, an emission feature at $4471 \AA$ is evident in the initial spectrum and absent in the quiescent spectra. During a flare, the amount of material emitting at chromospheric temperatures increases, and lines due to highexcitation species such as He I, which is responsible for this feature, appear (Reid \& Hawley 2005). The blue continuum visible in the initial spectrum is also typically observed in flare events (e.g., Hawley \& Pettersen 1991). In a forthcoming paper (E. Hilton et al. 2009, in preparation) we describe the properties of M stars for which we detected similar changes to the emission lines and continuum.
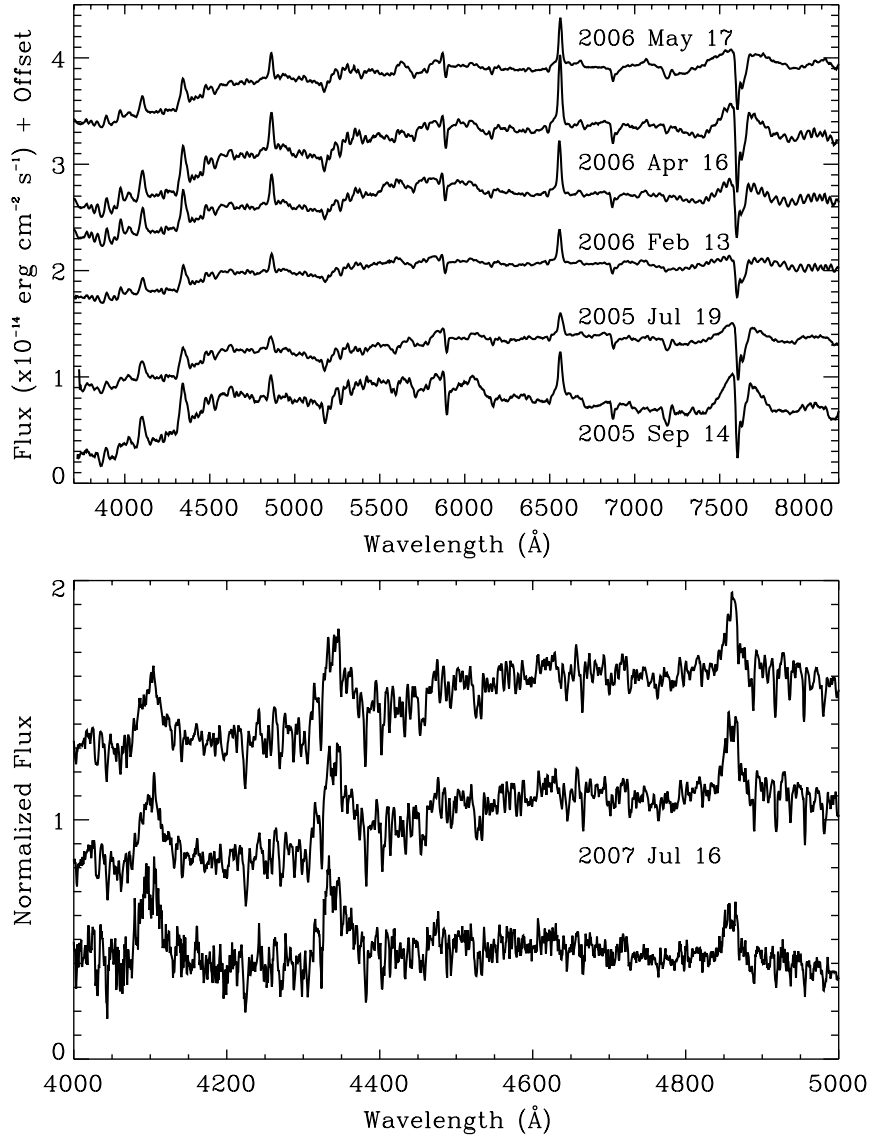

Figure 9. APO spectra of the proposed stellar counterpart to 1RXS J171456.2+585130. Top: low-resolution spectra, presented as in Figure 8. Two spectra were obtained on 2006 April 16. Bottom: high-resolution spectra of the region including the $\mathrm{H} \beta, \mathrm{H} \gamma$, and $\mathrm{H} \delta$ lines showing the structure typically seen in CVs.

\subsubsection{A New CV: SDSS J171456.78+585128.3}

APO spectra for the proposed counterpart to the RASS source RXS J171456.2+585130 are presented in Figure 9. We obtained six spectra for this star on five separate nights between 2005 September 14 and 2006 May 17 using DIS in the low-resolution setup. While the underlying spectrum corresponds to that of a K4 star, strong, persistent Balmer emission is obvious (see the top panel of Figure 9). The emission lines are broader than for the M star in Figure 8, suggesting that we are observing accretion rather than flaring, and there is therefore a currently invisible companion to the $\mathrm{K} 4$ star.

On 2007 July 16, we obtained three more spectra of this star using DIS in the high-resolution setup $\left(0.62 \AA \mathrm{pixel}^{-1}\right.$ in the blue, $0.56 \AA$ in the red, with the gratings centered on 4600 and $6800 \AA$, respectively). These spectra confirmed that the emission lines are broader than those of flaring stars, and also more structured than those typically seen in flares or in symbiotic systems (see bottom panel of Figure 9). We used IRAF's splot routine to measure the $\mathrm{H} \alpha$ and $\mathrm{H} \beta$ equivalent widths (EqWs) in our two longest observations (20 and 30 minutes). The average $\mathrm{H} \alpha \mathrm{EqW}=12.5 \AA$, corresponding to a flux of $9.5 \times 10^{-14} \mathrm{erg}$ $\mathrm{cm}^{-2} \mathrm{~s}^{-1}$; for $\mathrm{H} \beta$, the $\mathrm{EqW}=10.0 \AA$ and the flux $=7.0 \times 10^{-14}$ erg $\mathrm{cm}^{-2} \mathrm{~s}^{-1}$. We conclude that SDSS J1714 is a new CV.

To measure its period, we used DIS in its new low-resolution setup (1.9 $\AA \mathrm{pixel}^{-1}$ in the blue, $2.3 \AA$ in the red, with the gratings centered on 4295 and $6600 \AA$ ) to acquire a dozen 


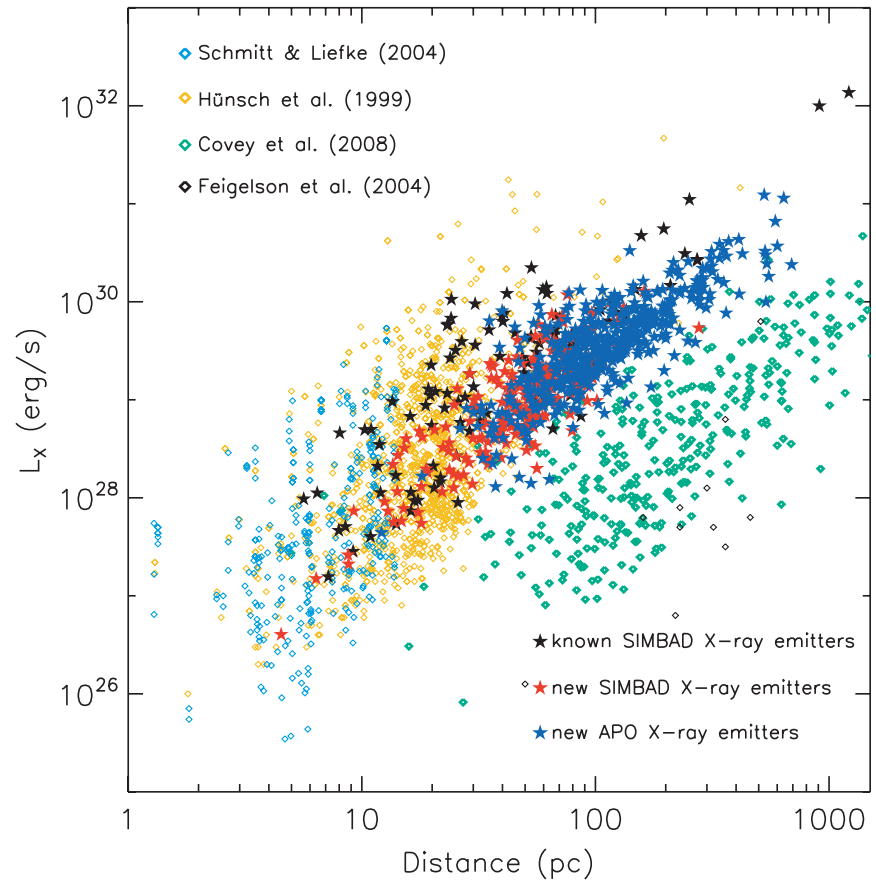

Figure 10. $L_{X}$ as a function of distance for our sample of X-ray-emitting stars. Also shown are the samples of Schmitt \& Liefke (2004), Hünsch et al. (1999), Covey et al. (2008), and Feigelson et al. (2004).

(A color version of this figure is available in the online journal.)

20 minute spectra on 2007 October 7. Since the secondary in this system is a $\mathrm{K}$ star, we expected the orbital period to be long, on the order of 5-6 hr. In fact, our radial velocity measurements suggest that the period is $\sim 10 \mathrm{hr}$ and further observations are required to determine SDSS J1714's period and nature. Its log $\left(f_{X} / f_{g}\right)$ of -1.44 , while low, is consistent with it being a DN, as is its position in the $\log \left(f_{X} / f_{g}\right)$ versus $\mathrm{H} \beta \mathrm{EqW}$ plot (see Section 6.1.1).

\section{THE CATALOG}

\subsection{Distances and X-ray Luminosities}

We derive distances to the F, G, K, and M stars in our catalog using photometric parallax relations appropriate for dwarfs on the main sequence, since these dominate our sample. We construct $B-K_{s}$ colors using the Tycho/USNO $B$ magnitudes for 662 stars in the catalog (48 lack a $B$ magnitude) and adopt the relationship between $g-K_{s}$ and $B-K_{s}$ derived by Covey et al. (2008). We then use a fit to the $\mathrm{M}_{K_{s}}$ versus $g-K_{s}$ tabulations of Kraus \& Hillenbrand (2007) to derive distances to each star. Adopting the same conversion of 1 count $\mathrm{s}^{-1}$ into $f_{X}=10^{-11} \mathrm{erg} \mathrm{cm}^{-2} \mathrm{~s}^{-1}$ used in Section 4.1.1, we calculate $\mathrm{X}$-ray luminosities for our stars.

These luminosities are shown in Figure 10 as a function of distance. We also plot data from several other catalogs of X-ray emitters. The Schmitt \& Liefke (2004) and Hünsch et al. (1999) catalogs are both of ROSAT detections of nearby stars, and the luminosities are also over the $0.1-2.4 \mathrm{keV}$ range. We also include the $11 \mathrm{X}$-ray-emitting stars identified by Feigelson et al. (2004) in the Chandra Deep Field North and the 348 identified by Covey et al. (2008) in the Extended Chandra Multiwavelength Project (here we use the 0.5-2.0 keV luminosities). Our sample covers a unique area in the $L_{X^{-}}$ distance plane, with $10^{27} \lesssim L_{X} \lesssim 10^{31} \mathrm{erg} \mathrm{s}^{-1}$ and $10 \lesssim \mathrm{D}$ $\lesssim 1000$ pc.

\subsection{Comparison to the Flesch \& Hardcastle (2004) and Parejko et al. (2008) Catalogs}

As a test of the robustness of our identifications, we queried the Flesch \& Hardcastle (2004) catalog for matches to our own. They provide classifications for $115 \mathrm{X}$-ray sources that appear in our catalog. ${ }^{24} 100$ have counterparts that are classified as stars; while these are therefore previously known sources, our work provides additional information (e.g., spectral types) not available in the Flesch \& Hardcastle (2004) catalog.

The 15 other sources have counterparts identified by Flesch \& Hardcastle (2004) as radio sources (six), galaxies (nine), or AGN/QSOs (three). However, in four of these cases, Flesch \& Hardcastle (2004) calculate that their catalog-level matching has most likely produced a random association between, e.g., a radio source and an optical object. For five sources, inspection of the SDSS data for the RASS error circles reveals that the position of the Flesch \& Hardcastle (2004) counterpart is that of our proposed stellar counterpart, or is offset but still consistent with being the star in our catalog (i.e., there is no SDSS object detected at the Flesch \& Hardcastle (2004) position and the nearest object is the bright star we identify as the source). The available SDSS data indicate that the proposed AGN/QSOs are the RASS source counterparts, and we remove these three sources from our catalog. This leaves only three cases in which Flesch \& Hardcastle (2004) propose a different (non-AGN) counterpart to the $\mathrm{X}$-ray source.

In another 171 cases, a source in our catalog is classified by Flesch \& Hardcastle (2004) simply as an X-ray source. They calculate that 39 of these entries most probably have stellar counterparts and that for another 50 sources their proposed association is most likely to be random. Inspection of the SDSS data for the remaining 82 Flesch \& Hardcastle (2004) RASS error circles finds that in 60 cases the counterpart's position is that of our cataloged star, or is offset but still consistent with being the star in our catalog. For 149 of these 171 sources, therefore, the Flesch \& Hardcastle (2004) classification either agrees with or does not contradict our own.

We also checked our catalog for entries listed in the Parejko et al. (2008) catalog of $\sim 1900$ X-ray-emitting galaxies, constructed from matching the RASS to the so-called main galaxy sample from the SDSS Data Release 4 (DR4; AdelmanMcCarthy et al. 2006). Thirty sources in our catalog are listed as having an SDSS galaxy as the X-ray counterpart. This sample is dominated by unclassifiable galaxies (24), which Parejko et al. (2008) define as galaxies for which most or all of their chosen diagnostic emission lines have fluxes too weak to be measured, and for which most of the matches to the RASS are in fact random. Unsurprisingly, only two of these galaxies are quoted as having a more than $50 \%$ chance of being the X-ray counterpart; 17 are less than $10 \%$ likely to be the RASS source. Of the six remaining galaxies, only a transition galaxy and a galaxy with unclassified emission, meaning that its position in the standard BPT diagrams (Baldwin et al. 1981) shifts according to the line ratios being considered, have a more than $50 \%$ chance of being the X-ray source counterpart.

Given this broad agreement between our classifications and those of both Flesch \& Hardcastle (2004) and Parejko et al. (2008) and the additional information provided by our catalog, we list all of the (non-AGN) sources that appear in their catalogs

\footnotetext{
24 In nine cases there are two entries in the Flesch \& Hardcastle (2004) catalog for what appear to be different detections of the same X-ray source.
} 
Table 4

Sample Data and Derived Quantities for the Stars in Our Catalog

\begin{tabular}{|c|c|c|c|c|c|c|c|c|c|c|}
\hline 1RXS J & $\begin{array}{c}\text { Pos. } \\
\text { Err. (") }\end{array}$ & Counts s $^{-1}$ & SDSS J & $\begin{array}{c}\text { Offset } \\
\left({ }^{\prime \prime}\right)\end{array}$ & $\begin{array}{c}\text { SDSS } g \\
(\mathrm{mag})\end{array}$ & $\begin{array}{c}\text { 2MASS } J \\
(\mathrm{mag})\end{array}$ & $\begin{array}{c}\log \\
\left(f_{X} / f_{J}\right)\end{array}$ & $\begin{array}{c}\text { Syn. } \mathrm{M}_{K_{s}} \\
(\mathrm{mag})\end{array}$ & $\begin{array}{c}\text { Distance } \\
(\mathrm{pc})\end{array}$ & $\begin{array}{c}L_{X} \\
\left(\mathrm{erg} \mathrm{s}^{-1}\right) \\
\end{array}$ \\
\hline $000053.1+002205$ & 23 & $0.025 \pm 0.011$ & $000051.96+002216.4$ & 20.5 & $12.79 \pm 0.00$ & $10.30 \pm 0.02$ & -2.18 & $4.63 \pm 0.08$ & $96 \pm 4$ & $2.8 \pm 1.2 \times 10^{29}$ \\
\hline $000312.8-001444$ & 26 & $0.025 \pm 0.011$ & $000312.63-001504.5$ & 20.2 & $14.37 \pm 0.02$ & $11.30 \pm 0.02$ & -1.78 & $4.72 \pm 0.09$ & $146 \pm 6$ & $6.4 \pm 2.7 \times 10^{29}$ \\
\hline $000447.9-095206$ & 23 & $0.051 \pm 0.015$ & $000446.97-095153.3$ & 19.0 & $11.66 \pm 0.00$ & $8.54 \pm 0.03$ & -2.58 & $4.45 \pm 0.07$ & & $1.6 \pm 0.5 \times 10^{29}$ \\
\hline $000659.3-000351$ & 13 & $0.023 \pm 0.009$ & $000658.40-000343.9$ & 15.4 & $12.59 \pm 0.00$ & $10.39 \pm 0.03$ & -2.18 & $4.63 \pm 0.08$ & $106 \pm 4$ & $3.1 \pm 1.2 \times 10^{29}$ \\
\hline $000714.2-103634$ & 12 & $0.062 \pm 0.016$ & $000714.06-103628.7$ & 5.6 & $12.60 \pm 0.00$ & $8.83 \pm 0.03$ & -2.38 & $5.89 \pm 0.16$ & $26 \pm 2$ & $5.1 \pm 1.3 \times 10^{28}$ \\
\hline $000803.1-002736$ & 15 & $0.041 \pm 0.012$ & $000803.42-002740.5$ & 6.6 & $15.92 \pm 0.02$ & $10.91 \pm 0.02$ & -1.73 & $7.17 \pm 0.36$ & $38 \pm 6$ & $7.1 \pm 2.2 \times 10^{28}$ \\
\hline $000921.2+003809$ & 10 & $0.114 \pm 0.019$ & $000921.79+003806.5$ & 9.4 & $12.09 \pm 0.00$ & $9.44 \pm 0.02$ & -1.87 & $4.68 \pm 0.08$ & & $5.6 \pm 0.9 \times 10^{29}$ \\
\hline $001745.3-010255$ & 12 & $0.097 \pm 0.019$ & $001745.03-010251.9$ & 5.0 & $10.34 \pm 0.00$ & $8.68 \pm 0.03$ & -2.24 & $3.94 \pm 0.18$ & $73 \pm 6$ & $6.2 \pm 1.2 \times 10^{29}$ \\
\hline $002127.8-110058$ & 17 & $0.057 \pm 0.017$ & $002127.08-110109.9$ & 15.5 & $12.63 \pm 0.00$ & $10.53 \pm 0.03$ & -1.73 & $4.45 \pm 0.07$ & $127 \pm 4$ & $1.1 \pm 0.3 \times 10^{30}$ \\
\hline $002552.2-095756$ & 22 & $0.055 \pm 0.016$ & $002550.97-095740.0$ & 24.4 & $14.80 \pm 0.03$ & $9.88 \pm 0.03$ & -2.01 & $\ldots$ & $\ldots$ & $\ldots$ \\
\hline
\end{tabular}

(This table is available in its entirety in a machine-readable form in the online journal. A portion is shown here for guidance regarding its form and content.)

and ours in our final catalog and note their Flesch \& Hardcastle (2004) and/or Parejko et al. (2008) classification.

\subsection{Available Data}

The full version of our catalog of 709 stars is available online only. Sample data and derived quantities are shown in Table 4. We give the RASS source name, positional error, and count rate, the SDSS counterpart's name, the offset between the X-ray and optical position, and the SDSS $g$ and 2MASS $J$ magnitudes. We caution that any SDSS photometry for objects brighter than $g \sim 14$ is suspect, and should be used only once the objects' flags (included in the full online catalog) have been considered. We then provide the calculated $\log \left(f_{X} / f_{J}\right)$, the synthetic $\mathbf{M}_{K_{s}}$ and corresponding distance for stars with a cataloged $B$, and the resulting X-ray luminosity and associated error (assuming 1 count $\mathrm{s}^{-1}$ corresponds to $f_{X}=10^{-11} \mathrm{erg} \mathrm{cm}^{-2} \mathrm{~s}^{-1}$ ).

The online catalog contains the complete SDSS and 2MASS photometry, and where relevant, SIMBAD data or APO observation dates and derived spectral types, as well as classification information from the Flesch \& Hardcastle (2004) or Parejko et al. (2008) catalog.

\subsection{Reliability of Our Identifications}

In order to test the reliability of our identifications, we replicate the test described in Section 3 to estimate the probability of false matches for the stars included in our final catalog. We shift the positions of our stars by a few arcminutes and rematch the catalog to the RASS. This consistently returns fewer than five matches, indicating that the chance of a random association between a star in our catalog and a RASS source is under $1 \%$.

A more conservative estimate of the number of false identifications in our catalog comes from our comparison to the Flesch \& Hardcastle (2004) catalog discussed above. There are roughly $300 \mathrm{X}$-ray sources listed in that catalog for which we also provide identifications. For $\sim 10 \%$ of those our classifications disagree, and we take this to be an upper limit on the contamination rate of our catalog as a whole.

\section{USING SDSS SPECTROSCOPIC CATALOGS TO IDENTIFY X-RAY EMITTING}

\subsection{Cataclysmic Variables}

As evidenced from their small numbers in our SIMBAD and APO sample of RASS counterparts, CVs and WDs (discussed in Section 6.2) are both rarer types of stellar X-ray sources. In
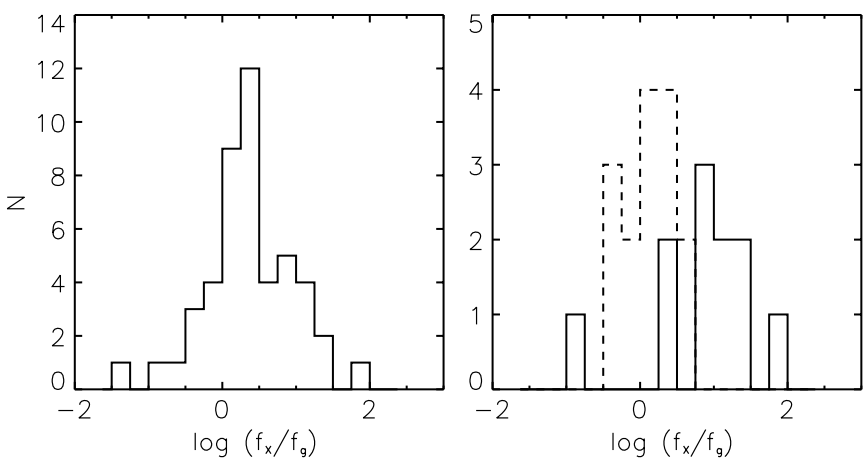

Figure 11. Left panel: distribution of $\log \left(f_{X} / f_{g}\right)$ for 47 SDSS CVs identified as RASS counterparts. Right panel: the same distribution for the known DNe (dashed histogram) and polars (solid histogram).

order to build up meaningful samples of these objects, we use the full SDSS spectroscopic catalogs for our correlations with the RASS.

The properties of the 177 new CVs spectroscopically identified in SDSS have been described by Szkody et al. (2002, 2003, 2004a, 2005, 2006, 2007); an additional 36 previously known $\mathrm{CV}$ s have been recovered in the survey data. 45 SDSS CVs have $\mathrm{X}$-ray counterparts in the RASS; all but one have been published in the Szkody et al. papers (SDSS J100515.38+191107.9 will be included in the next catalog). Below we discuss the $\left(f_{X} / f_{\text {opt }}\right)$ distributions for the X-ray-emitting $\mathrm{CV}$ s and reproduce the Patterson \& Raymond (1985) $\log \left(f_{X} / f_{\text {opt }}\right)$ versus $\mathrm{H} \beta \mathrm{EqW}$ plot for the 46 for which we have spectra.

\subsubsection{Flux Ratios and $H \beta$ Equivalent Widths}

We calculate $\log \left(f_{X} / f_{g}\right)$ for the 45 X-ray-emitting SDSS $\mathrm{CVs}$, for EI UMa, the only CV in our RASS/SIMBAD sample not included in the SDSS CV catalogs, and for SDSS J1714, the $\mathrm{CV}$ discovered in our APO spectroscopic survey. While this distribution is fairly symmetric about the median $(+0.38)$ (left panel, Figure 11), we find, as expected, an offset between the flux ratios for confirmed polars (median $\left.\log \left(f_{X} / f_{g}\right)=+0.99\right)$ and for DNe (median $\left.\log \left(f_{X} / f_{g}\right)=+0.13\right)$ (right panel, Figure 11).

Patterson \& Raymond (1985) described a correlation between $\left(f_{X} / f_{V}\right)$ and the $\mathrm{EqW}$ of the $\mathrm{H} \beta$ emission line, a proxy for the CV's overall emission strength. In Figure 12, we plot these two quantities for our CV sample. ${ }^{25}$ The $\mathrm{H} \beta \mathrm{EqW}$ are reported in

\footnotetext{
${ }^{25}$ The color-dependent difference between $g$ and $V$ is small: $g=V+0.05$ for a low-redshift QSO with $B-V=0.3$ (Fukugita et al. 1996).
} 


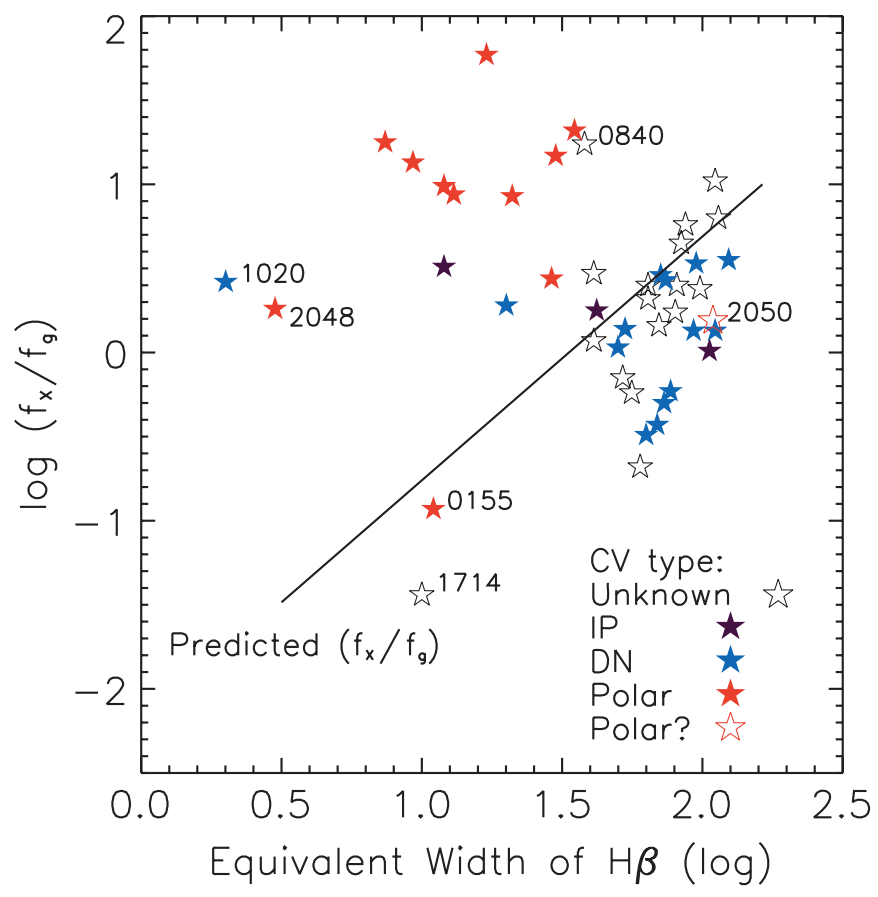

Figure 12. $\log \left(f_{X} / f_{g}\right)$ vs. EqWs of the $\mathrm{H} \beta$ line for the 46 X-ray-emitting SDSS CVs for which we have spectra. The predicted $\left(f_{X} / f_{g}\right)$ is from Patterson \& Raymond (1985). The labeled objects are discussed in the text.

(A color version of this figure is available in the online journal.)

Szkody et al. (2002, 2003, 2004a, 2005, 2006, 2007) for all but two of these CVs (SDSS J083642.80+532838.1/SW UMa and SDSS J100515.38+191107.9); in those cases (and that of our new CV) we measured them using IRAF's splot routine.

The scatter around the $\left(f_{X} / f_{g}\right)$ predicted by the Patterson \& Raymond (1985) relationship is comparable to that found in other studies (e.g., Richman 1996). However, there are clear departures from this relationship, with the polars appearing to form a distinct population from the $\mathrm{DNe}$ and other CVs. Furthermore, the SDSS CVs tend to have higher $\left(f_{X} / f_{\text {opt }}\right)$ ratio than those included in the earlier studies, where only a handful had $\log \left(f_{X} / f_{\text {opt }}\right) \geqslant 0$ (Richman 1996).

\subsubsection{Outliers}

SDSS J015543.40+002807.2 occupies an unusual position in Figure 12 for a polar because of its low X-ray-to-optical flux ratio $\left(\log \left(f_{X} / f_{g}\right)=-0.93\right)$. As noted by Szkody et al. (2002), SDSS J0155 was significantly brighter when observed by SDSS than in its Digitized Sky Survey image. A change in brightness of a few magnitudes-typical for polars entering a phase of stronger accretion-would be sufficient to explain the apparent inconsistency of this system's $\log \left(f_{X} / f_{g}\right)$ relative to that of other polars. Follow-up observations by Woudt et al. (2004) and Schmidt et al. (2005b) confirmed the large changes in mass transfer rate in this system.

The spectrum of SDSS J205017.84-053626.8 shows He II emission, but initial follow-up observations of this CV could not confirm that this is a magnetic system (Szkody et al. 2003). Its $\log \left(f_{X} / f_{g}\right)$ of +0.19 is similar to that of other intermediate polars (IPs) in our sample, but SDSS J2050 has a period of $1.6 \mathrm{hr}$, much shorter than is typical for IPs. Homer et al. (2006) determined that this is an example of the most magnetized type of $\mathrm{CV}$, a diskless, stream-fed polar.
SDSS J102026.53+530433.1, the DN KS UMa, has an anomalously low $\mathrm{H} \beta \mathrm{EqW}$ relative to other DNe; this is because its SDSS spectrum was taken during an outburst and is dominated by the accretion disk.

The spectrum of SDSS J204827.91+005008.9 is very unusual, with a hump apparent between 4000 and $5000 \AA$ and a typical M-dwarf continuum to the red of the hump (see Szkody et al. 2006). Follow-up observations by Schmidt et al. (2005a) led to the conclusion that this low-accretion magnetic binary is possibly a pre-polar. The $\mathrm{X}$-ray luminosity derived for this $\mathrm{CV}$ is an order of magnitude greater than the $L_{X}$ for similar SDSS systems, where the coronae of the late-type companion stars are thought to be responsible for the X-ray emission (Szkody et al. 2004b). Schmidt et al. (2005a) suggested that this weakens the case for SDSS J2048 as the ROSAT-detected X-ray source. Accordingly, on 2005 August 29 we obtained a DIS low-resolution spectrum for the most obvious potential X-ray counterpart in the RASS field, a bright nearby star $(g=14.43)$, which we then typed using the Hammer. This late G star has a 2MASS counterpart with $J=11.01$; the resulting $\log \left(f_{X} / f_{J}\right)$ of -2.15 is within the typical G star range (see Table 1). While this suggests that the G star may in fact be the RASS counterpart, this is a crowded field; pointed X-ray observations are required to confirm that this star, rather than SDSS J2048, is the RASS-detected $\mathrm{X}$-ray source.

Finally, when discovered, SDSS J084026.16+220446.6 did not appear to have strong He II emission (Szkody et al. 2006), but its $\log \left(f_{X} / f_{g}\right)$ and $\mathrm{H} \beta$ EqW are very similar to those of the known polar GG Leo. However, definitively classifying this system and many other SDSS CVs clearly requires more observational data than are provided by the survey.

\subsection{White Dwarfs}

WDs cool as they age, and the most commonly observed WDs, DAs, have an effective temperature $\left(T_{\text {eff }}\right)$ distribution that peaks at $\sim 10^{4} \mathrm{~K}$ (e.g., Eisenstein et al. 2006). At that temperature, one would not expect a typical DA to be a significant producer of thermal $\mathrm{X}$ rays. However, Shipman (1976) pointed out that the $\mathrm{X}$ rays produced in the hotter interior of a DA should travel through its thin hydrogen atmosphere unabsorbed.

The consensus was that the RASS would include upward of 5000 X-ray-emitting DAs (e.g., Barstow 1989), but to date the number found in the RASS is far smaller. Fleming et al. (1996) searched 55,000 RASS sources for the super-soft sources (HR $1=-1$ and HR $2 \leqslant 0$ ), found only 161 DAs, and concluded that $90 \%$ of DAs are opaque to X-ray emission. More recent RASS identification programs have added only a few dozen X-ray-emitting WDs to this sample (e.g., Zickgraf et al. 2003).

The SDSS DR4 produced a catalog of 9309 spectroscopically confirmed WDs. $\sim 8000$ of these are DAs and $\sim 6000$ are new (Eisenstein et al. 2006). Correlating SDSS WD lists with the RASS does not add many new WDs to the sample of known X-ray emitters (see Appendix $\mathrm{C}$ for details about our correlations). However, among these new X-ray emitters are three that, if confirmed, may be the coolest X-ray-emitting DAs detected (see Section 6.2.2), suggesting that these fainter SDSS $\mathrm{X}$-ray-emitting WDs, while small in number, may prove to be very interesting. Below we summarize our results before discussing the DAs' X-ray-to-optical flux ratio and temperature distributions. 
Table 5

New X-Ray-Emitting SDSS DA WDs

\begin{tabular}{|c|c|c|c|c|c|c|}
\hline SDSS J & $\begin{array}{l}T_{\text {eff }} \\
(\mathrm{K})\end{array}$ & 1RXS J & $\begin{array}{c}\text { Offset } \\
\left({ }^{\prime \prime}\right)\end{array}$ & Counts s ${ }^{-1}$ & $\begin{array}{c}\log \\
\left(f_{X} / f_{g}\right)\end{array}$ & $\begin{array}{l}\text { McCook \& } \\
\text { Sion (1999) }\end{array}$ \\
\hline $075106.51+301726.4$ & $33158 \pm 65$ & $075107.4+301719$ & 13.7 & $0.038 \pm 0.013$ & $-0.69^{a}$ & $\cdots$ \\
\hline $082346.14+245345.6$ & $34521 \pm 47$ & $082345.4+245344$ & 10.2 & $0.039 \pm 0.014$ & -0.81 & $\cdots$ \\
\hline $091215.43+011958.8$ & $55691 \pm 1415$ & $091215.5+012003$ & 4.8 & $0.041 \pm 0.015$ & $-0.01^{\mathrm{a}}$ & WD $0909+015$ \\
\hline $093006.79+522803.3$ & $36469 \pm 185$ & $093006.7+522752$ & 11.4 & $0.039 \pm 0.013$ & -0.53 & $\ldots$ \\
\hline $093608.90+382200.5$ & $37809 \pm 397$ & $093609.6+382237$ & 37.4 & $0.038 \pm 0.012$ & +0.00 & WD $0933+385$ \\
\hline $093921.83+264401.1^{b}$ & $59224 \pm 1253$ & $093922.8+264404$ & 13.3 & $0.044 \pm 0.013$ & -0.17 & $\ldots$ \\
\hline $095245.58+020938.9$ & $43114 \pm 443$ & $095244.6+020946$ & 16.6 & $0.025 \pm 0.010$ & -0.69 & WD $0950+023$ \\
\hline $100543.92+304744.9^{b}$ & $62299 \pm 1232$ & $100543.7+304809$ & 24.5 & $0.046 \pm 0.016$ & -0.42 & $\ldots$ \\
\hline $101339.56+061529.5$ & $14375 \pm 603$ & $101340.6+061556$ & 30.6 & $0.019 \pm 0.009$ & +0.70 & $\cdots$ \\
\hline $113836.33+475509.8$ & $50602 \pm 1169$ & $113836.9+475459$ & 12.3 & $0.046 \pm 0.015$ & +0.04 & $\cdots$ \\
\hline $120432.67+581936.9$ & $15725 \pm 545$ & $120428.0+581934$ & 36.9 & $0.022 \pm 0.010$ & +0.63 & $\cdots$ \\
\hline $125938.08+603900.0$ & $37640 \pm 225$ & $125936.6+603910$ & 14.8 & $0.039 \pm 0.012$ & -0.30 & $\cdots$ \\
\hline $132629.58+571131.5$ & $94382 \pm 5479$ & $132634.4+571052$ & 55.7 & $0.020 \pm 0.009$ & +0.11 & $\cdots$ \\
\hline $143736.67+362213.4^{b}$ & $\ldots$ & $143738.0+362237$ & 36.4 & $0.029 \pm 0.009$ & -0.23 & $\cdots$ \\
\hline $154448.25+455039.0$ & $53109 \pm 1119$ & $154449.3+455035$ & 11.5 & $0.046 \pm 0.011$ & -0.08 & $\ldots$ \\
\hline $163418.25+365827.1$ & $10080 \pm 216$ & $163421.8+365859$ & 53.0 & $0.016 \pm 0.007$ & +0.60 & $\cdots$ \\
\hline $022618.60-083049.3$ & $9692 \pm 153$ & $022615.8-083108$ & 45.8 & $0.033 \pm 0.011$ & +0.94 & WD $0223-087$ \\
\hline $074859.06+215445.7$ & $29717 \pm 911$ & $074858.9+215458$ & 12.5 & $0.026 \pm 0.010$ & +0.75 & $\ldots$ \\
\hline $093307.65+400637.9$ & $9037 \pm 57$ & $093305.6+400553$ & 50.7 & $0.035 \pm 0.011$ & +0.46 & $\cdots$ \\
\hline $093456.44+023121.1$ & $20844 \pm 1009$ & $093458.0+023151$ & 37.9 & $0.023 \pm 0.011$ & +0.60 & WD $0932+027$ \\
\hline $103237.35+512857.9$ & $20983 \pm 585$ & $103236.8+512809$ & 48.7 & $0.016 \pm 0.007$ & +0.35 & $\ldots$ \\
\hline $112740.90-024638.7$ & $20784 \pm 206$ & $112741.1-024614$ & 24.9 & $0.017 \pm 0.007$ & -0.45 & $\ldots$ \\
\hline $152349.89+041434.6$ & $23852 \pm 542$ & $152351.0+041356$ & 41.6 & $0.059 \pm 0.020$ & +0.49 & WD $1521+044$ \\
\hline $165645.49+182437.7$ & $12460 \pm 638$ & $165643.7+182441$ & 25.8 & $0.015 \pm 0.006$ & +0.66 & $\ldots$ \\
\hline $232658.89-002339.9$ & $8081 \pm 83$ & $232701.2-002337$ & 34.6 & $0.018 \pm 0.009$ & +0.37 & $\cdots$ \\
\hline $232659.23-002348.0$ & $10622 \pm 47$ & $232701.2-002337$ & 31.3 & $0.018 \pm 0.009$ & -0.37 & $\ldots$ \\
\hline
\end{tabular}

Notes. Above the line are highly secure RASS counterpart identifications; below it are tentative identifications requiring further observations. SDSS J0936 is listed as a DQ WD by McCook \& Sion (1999). However, the SDSS spectrum does not show the carbon absorption lines typical of DQs, and it seems that this is indeed a DA WD.

${ }^{a} g$ photometry is flagged in SDSS.

b Post-DR4 WD.

\subsubsection{New SDSS X-ray-Emitting White Dwarfs}

We list 17 new confident DA WD/RASS source associations in Table 5; this number includes three post-DR4 WDs, described below, and four McCook \& Sion (1999) DAs not previously identified as X-ray sources. ${ }^{26}$ Table 5 gives the relevant data for these DAs, including the McCook \& Sion (1999) catalog name if available.

Three of these DAs are not included in the Eisenstein et al. (2006) DR4 catalog: SDSS J093921.83+264401.1, $\mathrm{J} 100543.92+304744.9$, and $\mathrm{J} 143736.67+362213.4$. We have therefore fitted the SDSS spectra using the WD models described in Kleinman et al. (2004) to obtain estimates for their $T_{\text {eff }}$ and $\log g$. Because of calibration problems with the SDSS spectrum, this fitting fails for SDSS J1437, and we identify it as a DA based on visual inspection of the spectrum. For SDSS J0939, the fits yield that the star is a DA0.9 with $\log g=7.9$ and $T_{\text {eff }} \sim 59,000 \mathrm{~K}$; SDSS J1005 is a DA0.8 with $\log g=7.6$ and $T_{\text {eff }} \sim 62,000 \mathrm{~K}$ (see Appendix C3).

We also identify ten DAs as less-certain RASS counterparts and list them in Table 5 for reference. Follow-up optical and/or $\mathrm{X}$-ray observations are required to confirm these associations.

\footnotetext{
26 Our matching returned an additional 11 known X-ray-emitting DAs; ten were identified by Fleming et al. (1996) or Zickgraf et al. (2003), or both; the other DA was reported as an X-ray emitter by Chu et al. (2004).
}

We further identify several WDs of other types as RASS counterparts. Among these are three DAs with M star companions, two DB WDs, which have helium-rich atmospheres, and a PG 1159 star, a pre-WD star showing strong oxygen and carbon lines. We also identify two other non-DA WDs from our post-DR4 spectra as likely RASS sources. We list these objects in Table 6 and discuss follow-up Chandra observations of the two candidate X-ray-emitting DBs in Section 6.2.3.

\subsection{2. $\log \left(f_{X} / f_{g}\right)$ and Temperature Distributions}

In Figure 13, we plot the $\log \left(f_{X} / f_{g}\right)$ distribution for the 36 Fleming et al. (1996) X-ray-emitting DA WDs within the SDSS footprint. ${ }^{27}$ The median $\log \left(f_{X} / f_{g}\right)=-0.23$, with the $2 \sigma$ range being from -1.72 to +1.26 . The $20 \mathrm{WDs}$ not cataloged in Fleming et al. (1996) that we have confidently identified as Xray emitters have flux ratios that fall within this range. Although not included in this figure, the ten additional less-certain X-rayemitting DAs listed in Table 5 also have flux ratio values within this range.

In Figure 14, we compare the temperature distribution for our X-ray-emitting DAs to that of the 76 Fleming et al. (1996)

\footnotetext{
27 This includes the six WDs from Table 2 listed in the Fleming et al. (1996) catalog. Of the eight WDs in that table, only the last, WD 1725+586, has an SDSS spectrum. It is therefore the only X-ray-emitting WD identified in our earlier work included in the rest of this analysis.
} 
Table 6

X-Ray-Emitting SDSS Non-DA WDs

\begin{tabular}{lccrcccc}
\hline \hline & $\begin{array}{c}T_{\text {eff }} \\
\text { SDSS J }\end{array}$ & 1RXS J & $\begin{array}{c}\text { Offset } \\
\left({ }^{\prime \prime}\right)\end{array}$ & Counts s $^{-1}$ & $\begin{array}{c}\log \\
\left(f_{X} / f_{g}\right)\end{array}$ & $\begin{array}{c}\text { McCook \& } \\
\text { Type }\end{array}$ & Sion $(1999)$ \\
\hline $093122.86+362209.4$ & $20496 \pm 1050$ & $093119.2+362223$ & 46.4 & $0.017 \pm 0.008$ & +0.60 & DB & $\ldots$ \\
$153225.49+472700.9$ & $17567 \pm 447$ & $153221.7+472648$ & 40.4 & $0.012 \pm 0.005$ & +0.20 & DB & $\ldots$ \\
$114635.23+001233.5$ & $100000 \pm 794^{\mathrm{a}}$ & $114634.6+001238$ & 10.7 & $0.091 \pm 0.020$ & -0.72 & PG 1159 & $\ldots$ \\
$114312.57+000926.6$ & $17596 \pm 607$ & $114313.8+000955$ & 34.2 & $0.038 \pm 0.017$ & +0.22 & DA+dM6e & $\ldots$ \\
$132725.72+505711.2^{\mathrm{b}}$ & $10848 \pm 112^{\mathrm{a}}$ & $132725.7+505713$ & 2.0 & $0.047 \pm 0.012$ & +0.04 & DA+dM & WD 1325+512 \\
$134100.03+602610.4$ & $46306 \pm 0$ & $134101.0+602556$ & 15.7 & $0.034 \pm 0.009$ & -0.42 & DA+dM3e & $\ldots$ \\
$141740.23+130148.7^{\mathrm{b}}$ & $36000 \pm 51$ & $141741.2+130206$ & 22.5 & $0.032 \pm 0.012$ & -1.11 & DA+dM & WD 1415+132 \\
$223051.15+125706.8$ & $21125 \pm 880$ & $223051.6+125713$ & 9.4 & $0.023 \pm 0.010$ & +0.55 & DA+dM4e & $\ldots$ \\
\hline
\end{tabular}

Notes.

${ }^{a}$ Fit for $T_{\text {eff }}$ is unreliable.

${ }^{\mathrm{b}}$ Post-DR4 WD.

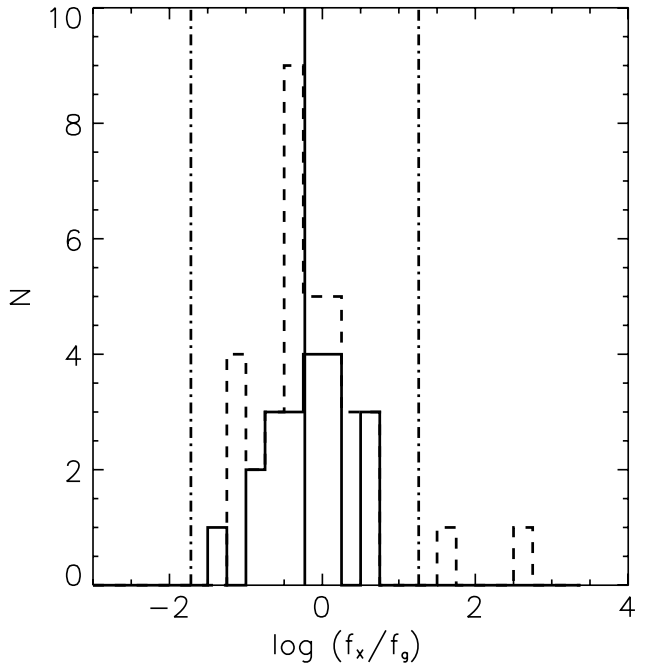

Figure 13. $\log \left(f_{X} / f_{g}\right)$ distributions for X-ray-emitting DA WDs. The dashed histogram is for the 36Fleming et al. (1996) DAs in the SDSS footprint; the solid one is for the sample identified here (eight recovered Fleming et al. (1996) DAs are not included in the latter histogram). The solid vertical line is the mean flux ratio, and the dot-dashed vertical lines bracket the $2 \sigma$ ranges, for the Fleming et al. (1996) sample.

DAs for which Marsh et al. (1997) obtained temperatures. We include 15 previously known and 12 new DAs for which the association with a RASS source is most secure. ${ }^{28}$ Only three Fleming et al. (1996) DAs in our sample have Marsh et al. (1997) temperatures and are plotted twice: WD $1026+453,1429+373$, and $1725+5863$. For these DAs, the difference between the Eisenstein et al. (2006) and Marsh et al. (1997) $T_{\text {eff }}$ are rather large $(2700,1900$, and $8900 \mathrm{~K})$, with the Eisenstein et al. (2006) $T_{\text {eff }}$ being greater in each case. Discrepancies such as these have been noticed in comparisons between SDSS temperatures and those in the literature, particularly for the hotter objects, with the differences reaching $\sim 10 \%$ at 50,000 K (D. Eisenstein 2006, private communication). Nevertheless, the temperature distributions for our sample of X-ray-emitting DAs (median $T_{\text {eff }}=43,100 \mathrm{~K}$ ) and that of Fleming et al. (1996) are very similar.

We also plot the $T_{\text {eff }}$ distribution for 7578 Eisenstein et al. (2006) DAs, normalized to the maximum of the Fleming et al. (1996) distribution. The separation between X-ray emitters and "ordinary" WDs is clear, with the Fleming et al. (1996) DAs

\footnotetext{
${ }^{28}$ One new DA, SDSS J1437, lacks a $T_{\text {eff }}$, as mentioned above.
}

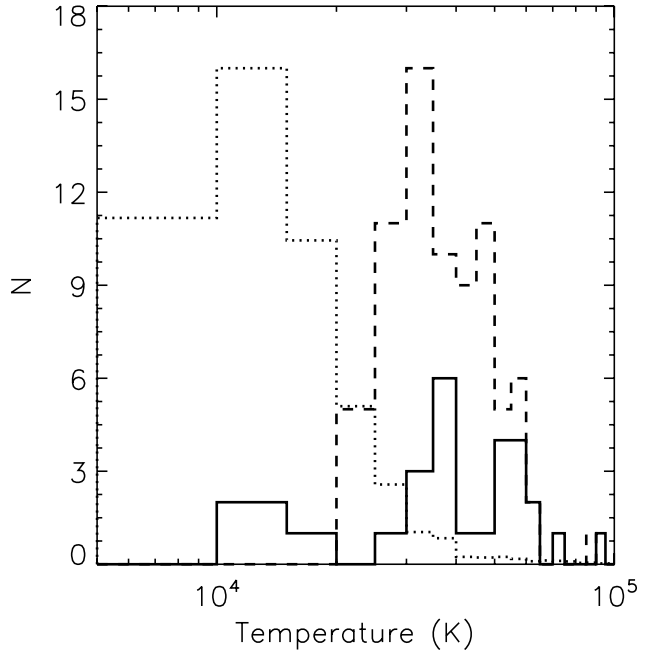

Figure 14. Temperature distributions for X-ray-emitting and "ordinary" DA WDs. The dashed histogram is the Fleming et al. (1996) sample of X-ray emitters; the solid one is the sample identified from SDSS/RASS matching. The dotted histogram is the normalized distribution for 7578 Eisenstein et al. (2006) DAs.

having a median $T_{\text {eff }} \sim 39,000 \mathrm{~K}$, compared to $14,000 \mathrm{~K}$ for the Eisenstein et al. (2006) DAs. Interestingly, 1251 of the Eisenstein et al. (2006) DAs are hotter than 22,153 K, the temperature of the coolest Fleming et al. (1996) DA. Even if, as suggested by Fleming et al. (1996), $90 \%$ of DAs are opaque to $\mathrm{X}$ rays, we would expect $\sim 125$ of these to be X-ray sources. If we include the uncertain WD/RASS associations, we have to date found roughly half that number. One possible explanation for this discrepancy is that the use of the Fleming et al. (1996) super-softness criterion is too restrictive at fainter X-ray fluxes, where the hardness ratios have larger errors. Alternatively, these sources may be too faint to be detected in the RASS. We plan to explore this question further.

Three of our new X-ray-emitting DAs are cooler than the coolest Fleming et al. (1996) DA and below the minimum temperature theoretically needed for X-ray emission from a DA. The three DAs, SDSS J101339.56 + 061529.5, $\mathrm{J} 120432.67+581936.9$, and $\mathrm{J} 163418.25+365827.1$, are faint $(g>19 \mathrm{mag})$, and the quoted $T_{\text {eff }}$ should be considered with caution. That said, because of the strength of the Balmer absorption lines, model fits to lower temperature SDSS WD spectra are very reliable (D. Eisenstein 2006, private communication). Follow-up optical and especially X-ray observations, given the 
low X-ray detection likelihoods of the RASS sources, are called for.

One of these DAs, SDSS J1634, was observed with XMM on 2008 Mar 11 (ObsId 0503550701). The X-ray background for these observations was very high; the on-source integration time was very short ( $2200 \mathrm{~s}$ rather than the $8000 \mathrm{~s}$ requested) and only MOS data are available. As the MOS is not very sensitive at soft energies, the nondetection of the WD in these data is neither surprising nor conclusive.

\subsubsection{Observations of Candidate Cool X-Ray-Emitting DBs}

The first two WDs listed in Table 6 are DB WDs newly identified by Eisenstein et al. (2006). These DBs are cooler than one would expect for non-DA X-ray emitters (see discussion in O'Dwyer et al. 2003); however, both are at relatively large offsets from the corresponding RASS sources. Furthermore, these RASS sources have low detection likelihoods; visual inspection of the RASS images suggests that both sources were marginally detected. $5 \mathrm{ks}$ Chandra snapshots of SDSS J0931 on 2008 January 16 (ObsId 8916) and J1532 on 2008 June 1 (ObsId 8917) were therefore taken to locate the RASS source and test whether the DBs are in fact X-ray sources. Sources were identified using the Ciao routine dmextract.

There is no Chandra source at the position of the WD in either observation, nor do we appear to have detected either of the RASS sources. We discuss each observation below; we conclude that the RASS sources were either spurious or transient.

1. SDSS J0931. The closest source to the RASS position is a weekly detected $(<3 \sigma)$ optically faint $(g=22.1 \mathrm{mag})$ galaxy. ${ }^{29}$ Taking into account the photometric errors, SDSS $\mathrm{J} 093119.70+362202.4$ has $u-g \lesssim 0.6$, giving it the UVexcess typically seen in SDSS QSOs (see Richards et al. 2002); its $\log \left(f_{X} / f_{g}\right)$ of 1.0 is consistent with this being an AGN or QSO. While it is offset by $22^{\prime \prime}$ from the RASS position, it seems unlikely that this is the RASS source, however, as its Chandra count rate is substantially less than that predicted from the RASS source count rate.

We note the serendipitous detection of the counterpart to the RASS source 1RXS J093210.1 + 363010. SDSS J093209.60 + 363002.5 is a relatively bright $(g=17.8 \mathrm{mag})^{30} z=0.15$ earlytype galaxy lacking both the photometric UV-excess and the strong spectroscopic emission lines typically seen in AGN. The likely counterpart to a FIRST source, this is plausibly an X-rayemitting BL Lac, as suggested by Plotkin et al. (2008), although it also has some similarities to e.g., CXOU J031238.9-765134, an X-ray bright optically normal galaxy (XBONG; Comastri et al. 2002).

2. SDSS J1532. The closest Chandra-detected source corresponds to a faint SDSS object ( $g=21.1 \mathrm{mag})$. Within the photometric errors, SDSS J153244.22+472405.2 has $u-g<0.6$; its $\log \left(f_{X} / f_{g}\right)$ of 0.8 is consistent with this being an AGN or QSO. However, this object is over $4^{\prime}$ from the RASS position and its Chandra count rate is significantly less than that predicted based on the RASS source count rate.

\section{CONCLUSION}

We have used photometric and spectroscopic data from SDSS to identify 709 stellar X-ray emitters detected in the ROSAT AllSky Survey. The majority of these are optically bright stars with

\footnotetext{
$29 \mathrm{Star} /$ galaxy separation at these magnitudes is unreliable (Scranton et al. 2002).

30 This is the Petrosian magnitude.
}

coronal X-ray emission, and were therefore not identified based on routine SDSS spectroscopy, which typically does not target $\lesssim 15$ mag objects. Instead, we used SDSS imaging photometry, correlations with 2MASS and other catalogs, and spectroscopy from the APO $3.5 \mathrm{~m}$ telescope to identify these stellar X-ray counterparts. Our sample of 707 X-ray-emitting F, G, K, and $\mathrm{M}$ stars characterized in this fashion is one of the largest $\mathrm{X}$ ray-selected samples of such stars. Our catalog also includes a known WD, WD $1725+586$, not previously identified as an X-ray source, as well as a new X-ray-emitting CV, SDSS $\mathrm{J} 171456.78+585128.3$, identified on the basis of follow-up APO observations. We estimate that our contamination rate by false matches between a RASS source and a star is $\lesssim 10 \%$.

In order to expand the sample of X-ray-emitting WDs and CVs, we also matched the SDSS spectroscopic catalogs of these objects (i.e., Eisenstein et al. 2006; Szkody et al. 2007) to the RASS. We identified 44 of the SDSS CVs published to date as RASS sources; roughly half are SDSS discoveries, with the others being previously known systems. We add a new X-rayemitting CV to this list; SDSS J1005 + 1911 is among the CVs spectroscopically identified since the last SDSS catalog (Szkody et al. 2007) and is a SDSS discovery.

Correlating the SDSS DR4 WD catalog of Eisenstein et al. (2006) with the RASS does not yield very many new entries in the catalog of known X-ray-emitting WDs. We identify 25 DA WDs included in Eisenstein et al. (2006) as RASS sources; 14 of these are new identifications (e.g., not included in the Fleming et al. (1996), Zickgraf et al. (2003), and/or Chu et al. (2004) catalogs of X-ray-emitting WDs). Examination of postDR4 spectroscopy allows us to identify three additional SDSSdiscovered DAs as RASS counterparts, but the total number of $\mathrm{X}$-ray-emitting WDs in SDSS remains small.

This dearth of new X-ray-emitting WDs may be a result of the relative sensitivities of the two surveys. Stellar counterparts to RASS sources tend to be very bright optically, and can be identified without requiring the depth of SDSS - while the fainter WDs discovered by SDSS are unlikely to be bright enough in the X-ray regime to have RASS detections (e.g., because they are too distant). However, among the new RASS WDs are three that, if confirmed, may be the coolest X-rayemitting DAs detected to date, suggesting that these fainter SDSS X-ray-emitting WDs, while small in number, may prove to be very interesting. In these cases, as in the cases of other, less-certain WD/RASS associations we propose, follow-up Xray and/or optical observations are necessary.

We thank the referee for comments that improved the paper. We are grateful to the APO time allocation committee at the University of Washington for providing us with the nights needed to complete our spectroscopic program. We thank the telescope operators at the observatory for their assistance with our observations. We also thank Michelle Cash, Nick Cowan, Lee Mannikko, Anil Seth, and Kristine Washburn for using some of their APO observing time to obtain data for this program. Finally, we thank Beth Willman for sharing her matching code. M. Agüeros is supported by an NSF Astronomy and Astrophysics Postdoctoral Fellowship under award AST-0602099; much of the work described here was made possible by a NASA Harriett G. Jenkins Predoctoral Fellowship. Further NASA support was provided to K. Covey through the Spitzer Space Telescope Fellowship Program, through a contract issued by the Jet Propulsion Laboratory, California Institute of Technology under a contract with NASA. We gratefully acknowledge Chandra 
Table 7

RASS/SIMBAD Class II, III, IV, and A Stars

\begin{tabular}{|c|c|c|c|c|c|c|}
\hline $1 \mathrm{RXS} \mathrm{J}$ & Counts s $^{-1}$ & Name & $\begin{array}{c}\text { Offset } \\
\left({ }^{\prime \prime}\right)\end{array}$ & $\begin{array}{c}V \\
(\mathrm{mag}) \\
\end{array}$ & $\begin{array}{c}\log \\
\left(f_{X} / f_{V}\right) \\
\end{array}$ & Type \\
\hline $012041.7+154141$ & $0.162 \pm 0.027$ & HD 8110 & 9.6 & 7.28 & -3.51 & G8IV \\
\hline $015332.2+124047$ & $0.028 \pm 0.010$ & HD 11520 & 34.6 & 7.06 & -4.36 & F6III \\
\hline $082929.4+453210$ & $0.040 \pm 0.011$ & BD+46 1404 & 3.0 & 10.14 & -2.97 & K0III \\
\hline $113615.1+662047$ & $0.018 \pm 0.007$ & CCDM J11362+6621AB & 5.29 & 7.91 & -4.20 & $\mathrm{~F} 5 \mathrm{IV} ; * *$ \\
\hline $141050.6+623121$ & $0.018 \pm 0.006$ & HD 124370 & 17.0 & 8.29 & -4.06 & F2IV \\
\hline $164532.0+422651$ & $0.044 \pm 0.010$ & HD 151445 & 1.6 & 8.15 & -3.73 & F5IV \\
\hline $165947.2+375045$ & $0.028 \pm 0.008$ & HD 153777 & 35.2 & 8.47 & -3.79 & F2IV \\
\hline $171558.3+270804$ & $0.026 \pm 0.008$ & HD 156362 & 8.1 & 6.58 & -4.58 & K2III \\
\hline $171701.2+264252$ & $0.032 \pm 0.009$ & HD 156536 & 8.3 & 7.51 & -4.13 & F3IV \\
\hline $013552.3+000559$ & $0.025 \pm 0.010$ & HD 9806 & 17.7 & 9.39 & -3.48 & A2 \\
\hline $032728.1-065748$ & $0.063 \pm 0.015$ & HD 21468 & 5.2 & 9.07 & -3.20 & $\mathrm{~A} 2$ \\
\hline $093642.4+565820$ & $0.175 \pm 0.019$ & HD 82861 & 8.4 & 7.06 & -3.56 & $\mathrm{Am}$ \\
\hline $130412.8+031257$ & $0.027 \pm 0.012$ & HD 113498 & 36.3 & 9.55 & -3.37 & A2 \\
\hline $132218.6+055348$ & $0.039 \pm 0.016$ & $\mathrm{~V} * \mathrm{BC}$ Vir & 53.3 & 11.9 & -2.28 & $\mathrm{~A} ; \mathrm{RR} *$ \\
\hline $140251.4+614051$ & $0.012 \pm 0.005$ & HD 122944 & 32.1 & 7.30 & -4.64 & A2 \\
\hline $144952.6+035517$ & $0.020 \pm 0.011$ & HD 130770 & 14.5 & 8.29 & -4.01 & A3 \\
\hline $155108.2+525439$ & $0.118 \pm 0.014$ & HD 142282 & 21.9 & 6.5 & -3.96 & $\mathrm{~A} 0 ; * \mathrm{i} *$ \\
\hline $163147.7+453527$ & $0.041 \pm 0.009$ & $\mathrm{BD}+452422 \mathrm{~B}$ & 14.0 & 8.89 & -3.46 & $\mathrm{~A} 0 ; * \mathrm{i} *$ \\
\hline $165344.9+630608$ & $0.017 \pm 0.003$ & HD 153204 & 18.4 & 8.55 & -3.98 & A2 \\
\hline $171337.2+304151$ & $0.011 \pm 0.005$ & $\mathrm{~V} * \mathrm{TU} \mathrm{Her}$ & 50.8 & 11.2 & -3.09 & $\mathrm{~A} 5 ; \mathrm{Al} *$ \\
\hline
\end{tabular}

Notes. “**” or “*i*” indicates a double or multiple star system, "RR*” an RR Lyrae star, and "Al*” an Algol-type binary.

grants GO6-7058X and GO8-9012X for support of portions of our program.

This research has made use of data obtained from the Chandra Data Archive and software provided by the Chandra X-ray Center (CXC) in the application packages CIAO, ChIPS, and Sherpa. Some of this work is based on observations obtained with XMM-Newton, an ESA science mission with instruments and contributions directly funded by ESA Member States and NASA.

Funding for the SDSS and SDSS-II has been provided by the Alfred P. Sloan Foundation, the Participating Institutions, the National Science Foundation, the U.S. Department of Energy, the National Aeronautics and Space Administration, the Japanese Monbukagakusho, the Max Planck Society, and the Higher Education Funding Council for England. The SDSS Web Site is http://www.sdss.org/.

The SDSS is managed by the Astrophysical Research Consortium for the Participating Institutions. The Participating Institutions are the American Museum of Natural History, Astrophysical Institute Potsdam, University of Basel, University of Cambridge, Case Western Reserve University, University of Chicago, Drexel University, Fermilab, the Institute for Advanced Study, the Japan Participation Group, Johns Hopkins University, the Joint Institute for Nuclear Astrophysics, the Kavli Institute for Particle Astrophysics and Cosmology, the Korean Scientist Group, the Chinese Academy of Sciences (LAMOST), Los Alamos National Laboratory, the Max-PlanckInstitute for Astronomy (MPIA), the Max-Planck-Institute for Astrophysics (MPA), New Mexico State University, Ohio State University, University of Pittsburgh, University of Portsmouth, Princeton University, the United States Naval Observatory, and the University of Washington.

This publication makes use of data products from the Two Micron All Sky Survey, which is a joint project of the University of Massachusetts and the Infrared Processing and Analysis Center/California Institute of Technology, funded by the National Aeronautics and Space Administration and the National Science Foundation.

\section{APPENDIX A}

\section{NON-MAIN SEQUENCE AND A STARS}

Table 7 gives the basic properties of the nine SIMBAD stars within $1^{\prime}$ of a RASS source cataloged as belonging to luminosity classes II, III, and IV that have not previously been associated with an X-ray source. We recovered an additional 14 previously identified X-ray-emitting members of these luminosity classes (from Fleming et al. 1989a; Haisch \& Schmitt 1994; Favata et al. 1995; Hempelmann et al. 1996; Appenzeller et al. 1998; Hünsch et al. 1998a, 1998b).

Table 7 also includes these data for the 11 unpublished RASS/ SIMBAD A stars. A stars are not generally predicted to be $\mathrm{X}$-ray emitters; an unseen, young, active later-type companion is usually thought to be the source for the observed $\mathrm{X}$ rays (see discussion in Güdel 2004). While we recover seven A stars that are previously known X-ray sources (from Stocke et al. 1991; Simon et al. 1995; Shaw et al. 1996; Appenzeller et al. 1998; Hünsch et al. 1998b; Schröder \& Schmitt 2007), it is unlikely, therefore, that all the other matched A stars are X-ray sources. However, the available SDSS data do not allow us to propose other counterparts to these X-ray sources. Follow-up X-ray and/ or optical observations are required to test whether they are in fact the RASS sources.

In addition, we identified two candidate A star counterparts to RASS sources in our APO spectroscopic campaign. In both cases, however, the A star seems unlikely to be the RASS source. TYC 3071-1446-1, listed in SIMBAD without a spectral type, appears to be in front of a cluster of galaxies that includes 2MASX J16571256+3920176, identified as the RASS source RXS J165711.6+ 392028 .

The SDSS spectrum of SDSS J095220.07+531455.6, 33".5 away from RXS J095223.8 +531455, is that of a $z=0.12$ QSO. While the candidate stellar counterpart is closer $(19.2)$, the RASS hardness ratios $(\mathrm{HR} 1=-0.05 \pm 0.34$; HR2 $=$ $-0.15 \pm 0.46)$ are consistent with those commonly found for AGN (e.g., Voges et al. 1999), and it therefore seems most likely that the QSO is in fact the RASS source. 
Table 8

SIMBAD Stars with APO Spectra

\begin{tabular}{|c|c|c|c|c|c|c|c|}
\hline $1 \mathrm{RXS} \mathrm{J}$ & SDSS J & $\begin{array}{c}\text { Offset } \\
\left({ }^{\prime \prime}\right)\end{array}$ & $\begin{array}{l}\text { SIMBAD } \\
\text { name }\end{array}$ & $\begin{array}{c}\text { Offset } \\
\left({ }^{\prime \prime}\right)\end{array}$ & $\begin{array}{l}\text { Published } \\
\text { Type }\end{array}$ & $\begin{array}{c}\text { First APO } \\
\text { Observation }\end{array}$ & $\begin{array}{l}\text { APO } \\
\text { Type }\end{array}$ \\
\hline $002552.2-095756$ & $002550.97-095740.0$ & 24.4 & NLTT 1354 & 0.2 & M3 & 2003 Sep 03 & M4 \\
\hline $010257.2-095145$ & $010257.42-095140.5$ & 8.8 & HD 6204 & 0.1 & G0 & 2003 Sep 03 & G3 \\
\hline $012724.2-093359$ & $012723.80-093409.1$ & 11.3 & FBS 0124-098 & 0.1 & M3... & 2003 Nov 08 & M3 \\
\hline $020000.4+003216$ & $015959.78+003219.3$ & 9.8 & [BHR2005] 284 & 0.4 & M3.5V & 2003 Nov 16 & M3 \\
\hline $020144.5-101743$ & $020143.87-101728.9$ & 16.8 & NLTT 6782 & 0.3 & M3... & 2003 Nov 08 & M4 \\
\hline $020221.0-010715$ & $020223.99-010708.3$ & 45.4 & BD-01 277 & 0.4 & K0 & 2005 Nov 20 & K3 \\
\hline $021042.8-093817$ & $021044.57-093826.0$ & 27.7 & NLTT 7231 & 0.0 & M4 & 2003 Nov 08 & M3 \\
\hline $021559.9-092913$ & $021558.94-092912.4$ & 14.1 & EUVE J0215-09.5 & 2.5 & $\mathrm{Me}$ & 2004 Oct 04 & M3 \\
\hline $022839.4-093550$ & $022839.68-093545.5$ & 6.5 & HD 15424 & 0.3 & F8 & 2005 Jan 05 & $\mathrm{~F} 2$ \\
\hline $022942.6-003026$ & $022941.79-003029.2$ & 12.5 & BD-01 341a & 1.0 & $\mathrm{G}$ & 2003 Sep 03 & G8 \\
\hline $025150.0-081338$ & $025148.21-081342.3$ & 26.8 & FBS 0249-084 & 0.3 & M5... & 2004 Oct 04 & M4 \\
\hline $031114.2+010655$ & $031115.49+010630.8$ & 31.4 & [BHR2005] 344 & 0.2 & M5.5V & 2005 Nov 20 & M6 \\
\hline $101627.8-005127$ & $101626.93-005139.4$ & 17.6 & RX J1016.4-0051 & 2.4 & M0e & 2004 Dec 03 & M1 \\
\hline $103546.1+021559$ & $103546.90+021558.3$ & 12.1 & $\mathrm{RX} 1035.7+0215^{\mathrm{b}}$ & 1.1 & M2e & 2004 Dec 03 & M3 \\
\hline $104743.9+652750$ & $104743.71+652736.0$ & 14.1 & HD 93270 & 0.3 & F8 & 2005 Jan 29 & F7 \\
\hline $115749.7+663406$ & $115749.80+663344.1$ & 21.9 & GSC 04160-00286 & 0.3 & M & 2004 Dec 03 & M4 \\
\hline $142049.4+604930$ & $142049.51+604934.9$ & 4.5 & RX J1420.8+6049c & 3.9 & M & 2004 Apr 30 & M3 \\
\hline $144243.9-003955$ & $144244.40-003955.4$ & 7.6 & $\mathrm{BD}-002862^{\mathrm{d}}$ & 0.3 & $\mathrm{G} 5 \mathrm{~V}+\mathrm{K} 1 \mathrm{~V}$ & 2005 Mar 01 & G2 \\
\hline $152346.8-004434$ & $152346.11-004424.8$ & 14.2 & TYC $5003-309-1^{\mathrm{e}}$ & 1.7 & $\mathrm{Ke}$ & 2005 Mar 30 & K1e \\
\hline $153826.3+523407$ & $153826.38+523418.2^{\mathrm{f}}$ & 11.3 & HD 234250 & 1.7 & $\mathrm{~K} 2$ & 2005 Apr 26 & $\mathrm{~K} 3$ \\
\hline $153912.0+554357$ & $153912.04+554355.2$ & 1.6 & HD 238488 & 0.4 & F8 & 2005 Apr 26 & F8 \\
\hline $165110.5+355500$ & $165109.95+355507.2$ & 9.5 & NLTT 43695 & 0.2 & M4 & 2005 May 31 & M5 \\
\hline $165445.3+423237$ & $165445.04+423227.5$ & 9.9 & RX J1654.7 + 4232 & 0.1 & M & 2005 May 31 & M3 \\
\hline $170352.9+321147$ & $170352.87+321146.2$ & 0.9 & TYC $2594-1478-1^{\mathrm{a}}$ & 0.6 & M2.5 & $2005 \mathrm{Jul} 19$ & M3 \\
\hline $172142.4+620036$ & $172142.60+620032.8$ & 4.0 & RX J1721.7 + 6200g & 4.0 & K4 & 2005 Sep 14 & $\mathrm{~K} 2$ \\
\hline $172439.2+644054$ & $172438.98+644052.5$ & 2.0 & TYC $4206-1161-1^{\mathrm{g}}$ & 0.1 & G0 & $2005 \mathrm{Jul} 20$ & G7 \\
\hline $230240.3+003453$ & $230241.41+003450.2$ & 17.0 & {$[\mathrm{ZEH} 2003] \mathrm{RX} \mathrm{J} 2302.6+00343^{\mathrm{c}}$} & 1.3 & $\mathrm{~K}$ & 2005 Nov 09 & $\mathrm{~K} 7 \mathrm{e}$ \\
\hline $074553.1+355818$ & $074555.71+355836.0$ & 36.5 & HD 62547 & 2.4 & K0 & 2005 Jan 05 & G7 \\
\hline $081546.8+460123$ & $081548.99+460119.5$ & 23.2 & [ZEH2003] RX J0815.7+4601 $1^{\mathrm{c}}$ & 3.2 & $\mathrm{~F} / \mathrm{G}$ & 2005 Jan 29 & M2 \\
\hline $091609.6+015320$ & $091610.17+015308.8$ & 14.1 & [ZEH2003] RX J0916.1+0153 3 & 3.4 & $\mathrm{~K}$ & 2005 Jan 29 & M4 \\
\hline $092023.1+021351$ & $092023.40+021340.5$ & 11.4 & [ZEH2003] RX J0920.3+0213 $1^{\mathrm{c}}$ & 0.5 & $\mathrm{~F} / \mathrm{G}$ & 2005 Jan 29 & $\mathrm{~K} 7 \mathrm{e}$ \\
\hline $101716.7+051145$ & $101716.76+051149.8$ & 4.4 & {$[\mathrm{ZEH} 2003] \mathrm{RX} \mathrm{J} 1017.2+05112^{\mathrm{c}}$} & 1.0 & $\mathrm{~F} / \mathrm{G}$ & 2005 Jan 29 & G5 \\
\hline $102547.0+033143$ & $102545.64+033141.0$ & 21.0 & {$[\mathrm{ZEH} 2003] \mathrm{RX} \mathrm{J} 1025.7+03312^{\mathrm{c}}$} & 0.7 & $\mathrm{~F} / \mathrm{G}$ & 2004 Mar 28 & $\mathrm{G} 2$ \\
\hline $105706.3-010109$ & $105707.70-010115.5$ & 21.9 & RX J1057.1-0101 & 3.4 & $\mathrm{~K} 4 \mathrm{~V}$ & 2004 Dec 03 & G5 \\
\hline $110046.1+052307$ & $110046.08+052304.6$ & 2.4 & {$[\mathrm{ZEH} 2003] \mathrm{RX} \mathrm{J} 1100.7+05231^{\mathrm{c}}$} & 1.5 & $\mathrm{~K}$ & 2004 Mar 28 & M3 \\
\hline $110804.1+052216$ & $110803.94+052214.3$ & 2.9 & $\mathrm{RX} \mathrm{J} 1108.0+0522^{\mathrm{c}}$ & 2.9 & $\mathrm{~F} / \mathrm{G}$ & 2004 Mar 28 & M0 \\
\hline $143139.0+004732$ & $143138.92+004731.4$ & 1.5 & 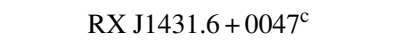 & 2.7 & $\mathrm{~K}$ & 2005 Mar 01 & M0 \\
\hline $153709.0+531928$ & $153709.43+531920.3$ & 9.0 & StKM 1-1262 & 2.7 & K5 & 2005 Feb 26 & M0 \\
\hline $160602.0+501124$ & $160602.24+501113.0$ & 11.7 & $\mathrm{~V} * \mathrm{~V} 842 \mathrm{Her}$ & 0.3 & F9V & 2005 Apr 26 & $\mathrm{G} 2$ \\
\hline $161022.6+450934$ & $161022.24+450934.9$ & 3.8 & RX J1610.3+4509 & 4.9 & $\mathrm{~K}$ & 2004 May 22 & M0 \\
\hline $171928.8+652227$ & $171928.68+652229.7$ & 2.4 & RX J1719.4+6522g & 2.4 & K0 & 2005 May 31 & G7 \\
\hline
\end{tabular}

Notes. The first offset is between the RASS and SDSS positions; the second is between the SDSS and SIMBAD positions. Stars above the line are those for which choosing the APO or the SIMBAD spectral class does not impact $\left(f_{X} / f_{\text {opt }}\right)$ calculations.

${ }^{a}$ X-ray source identified by Schwope et al. (2000).

b X-ray source identified by Appenzeller et al. (1998).

c X-ray source identified by Zickgraf et al. (2003).

${ }^{\mathrm{d}}$ X-ray source identified by Metanomski et al. (1998).

e X-ray source identified by Fischer et al. (1998).

${ }^{\mathrm{f}}$ One of two possible counterparts to this source (see Table 3). This star was not included in the flux ration calculations discussed in Section 4.1.1.

g X-ray source identified by Gioia et al. (2003).

$\mathrm{h}$ APO target is not the same star.

\section{APPENDIX B}

\section{COMPARISON OF SIMBAD AND APO STELLAR CLASSIFICATIONS}

In Table 8, we list all the SIMBAD-cataloged candidate RASS counterparts with a spectral type for which we obtained an APO spectrum. For 27 stars the spectral type derived from our spectrum either does not differ significantly from the SIMBAD type or is within the same spectral class. In these cases choosing one type or the other has no impact on the flux ratio calculations, since e.g., all F stars are binned together. In 15 of the 20 cases where subtypes are available in SIMBAD, the DIS-derived and SIMBAD spectral classes agree to within two subtypes. (The DIS spectra allow us to assign a subtype to seven other stars for which none was available.)

For another 14 stars, the choice of stellar type does impact the flux ratio calculations. In nine cases the star's type was derived by Zickgraf et al. (2003) from low-resolution spectra; we consider our types, obtained from higher-resolution spectra, 
to be more reliable. In the five other cases, a stellar subtype is listed in SIMBAD; our DIS-derived spectral type agrees to within three subtypes in four cases-only slightly worse than what we consider our typical uncertainty when typing with the Hammer. The fourth case is that of the counterpart to 1RXS J105706.3-010109, identified by Appenzeller et al. (1998) as the K4V star RX J1057.1-0101. Our APO target was a star offset by $\sim 5^{\prime \prime}$ from this one, explaining the discrepancy in spectral types.

Twenty two of these stars are previously identified X-ray sources; three additional stars are listed in SIMBAD as RASS counterparts, but the origin of these associations is unclear.

\section{APPENDIX C}

\section{SDSS WD/RASS IDENTIFICATIONS}

\section{Building the Sample of X-Ray-Emitting DAs}

Table 5 lists our most confident SDSS DA/RASS associations. We discuss how we obtained these and our less-certain identifications. Ten of these less-certain cases are also listed in Table 5; follow-up optical and/or X-ray observations are required to confirm these identifications.

\subsection{DAs Discovered Before SDSS}

In correlating the Eisenstein et al. (2006) catalog with the RASS, we identified 22 DAs listed in the McCook \& Sion (1999) catalog with an X-ray source within $1^{\prime}$. We classified seven of these sources as unlikely X-ray-emitting WDs. In four cases, a known QSO is in the RASS error circle and the X-ray source is not super-soft, which is consistent with the QSOs being the X-ray sources.

In three ambiguous cases, the sources are not super-soft and at large separations from the RASS source, but the available SDSS data do not rule out the DAs as X-ray sources. SDSS J022618.60-083049.3 is in a RASS error circle with two bright stars $(g=12.98$ and 13.19). The RASS source is not super-soft $(\mathrm{HR} 1=0.22 \pm 0.36)$ and it is therefore unlikely that the WD is the X-ray source. SDSS J093456.44+023121.1 is in a error circle with no obvious X-ray source counterparts. There is a very faint, red star closer to the RASS position $(g=21.35, r=$ 20.06), but were it the X-ray counterpart, its $\log \left(f_{X} / f_{g}\right)$ would be much higher than typical for stars. The RASS source is not soft (HR1 = 0.36 \pm 0.52$)$, so perhaps this is a QSO with stellar colors. Finally, SDSS J152349.89+041434.6 is in a error circle with SDSS J152349.88+041400.8, a $g=17.71$ galaxy. In these cases follow-up spectroscopy is needed to reveal, for example, whether this galaxy hosts an AGN. We consider these three WDs to be less-certain RASS counterparts and list them as such in Table 5.

Of the remaining 15 previously known DAs, we include in Table 5 the four that have not been identified as X-ray emitters (i.e., they are not in the Fleming et al. (1996), Zickgraf et al. (2003), or Chu et al. (2004) catalogs) as new identifications.

\subsection{SDSS DR4-Discovered DAs}

Our SDSS/RASS correlations yielded a further 25 SDSSdiscovered DA WDs (i.e., not listed in the McCook \& Sion (1999) catalog) with an X-ray source within $1^{\prime}$. Five of these sources also have nearby cataloged QSOs and are not super-soft. In three other cases we identify plausible non-WD counterparts to the RASS sources. A UV-excess source with $u-g=0.00 \pm$
0.03 is positionally coincident with the RASS source for which SDSS J083300.12 + 272254.5 is proposed as a counterpart. Similarly, another UV-excess source (with $u-g=0.24 \pm 0.13$ ) is closer than SDSS J094437.72 + 493815.3 to the RASS source for which the WD is the candidate counterpart. Finally, for SDSS J162814.59 + 363008.3 a very faint UV-excess object ( $g=21.45, u-g=0.18 \pm 0.13$ ) is positionally coincident with a FIRST radio source. In all three cases the RASS sources are not super-soft, and it seems likely that the UV-excess objects are QSOs and X-ray sources. Follow-up spectroscopy is required to confirm this, but we consider these DAs to be unlikely RASSsource counterparts.

Of the 17 remaining DAs, there are seven cases in which inspection of the RASS error circle, combined with the RASS source hardness ratio, suggests that the WD is probably not the X-ray source-but does not provide a strong alternate X-ray counterpart candidate. In all these error circles spectroscopy of multiple objects or high spatial resolution X-ray observations are needed. We consider these seven WDs to be less-certain RASS counterparts and list them as such in Table 5.

The remaining ten SDSS DR4 DAs are listed in Table 5 as new identifications.

\section{Building the Sample of X-Ray-Emitting non-DAs}

Our SDSS/RASS correlations also returned 11 non-DA WDs. Once again we eliminate those WDs in error circles with cataloged QSOs; there are three such error circles here, all with RASS sources that are not super-soft. For two other RASS sources the SDSS data suggest other counterparts. SDSS J123337.71+570335.4 is a $z=0.351$ redshift QSO nearer to the RASS source (HR1 $=0.20 \pm 0.42$ ) than SDSS $\mathrm{J} 123345.52$ + 570343.9, a DB WD. The UV-excess object SDSS J155209.12 + $433821.8(u-g=0.45 \pm 0.04)$ is also closer to the RASS source (HR1 $=-0.18 \pm 0.37$ ) than SDSS $\mathrm{J} 155205.59+433825.8$, one of the DA+M-star binaries. While spectroscopic confirmation is needed in the latter case, we conclude that both of these RASS sources are QSOs.

The six remaining WDs are the likely RASS sources listed in Table 6. In addition to the two DBs discussed in Section 6.2.3, these objects include the previously known PG 1159 star SDSS J114635.23+001233.5. The spectrum for this (very hot) star is poorly fit by our models, and the value for $T_{\text {eff }}$ in Table 6 should therefore not be trusted. The five other stars are DA+M-star systems, of which one is an SDSS discovery, SDSS J223051.15+125706.8 (Silvestri et al. 2006). For the three systems included in the Silvestri et al. (2006) DR4 catalog of close binary systems, we give the published $T_{\text {eff }}$ and note the $\mathrm{M}$ stars' spectral types. For the two other systems, SDSS J132725.72+505711.0 (WD $1325+512$ ) and $\mathrm{J} 141740.22+130148.5$ (WD $1415+132$ ), we fit the spectra to obtain $T_{\text {eff }}$ (listed in Table 6) and $\log g(8.93 \pm 0.09$ and $7.19 \pm 0.02$, respectively). The fit to the spectrum of SDSS $\mathrm{J} 1327$ is poor; the spectrum has relatively low signal to noise, and the presence of the $\mathrm{M}$ dwarf appears to have corrupted the fit to the $\mathrm{H} \alpha$ line.

\section{Building a Sample of X-Ray-Emitting WDs Identified Since DR4}

Since the production of the Eisenstein et al. (2006) WD catalog, new WDs have been identified through inspection of the more recent SDSS spectroscopy. We have also collected a few objects whose spectra were taken earlier in the survey but 

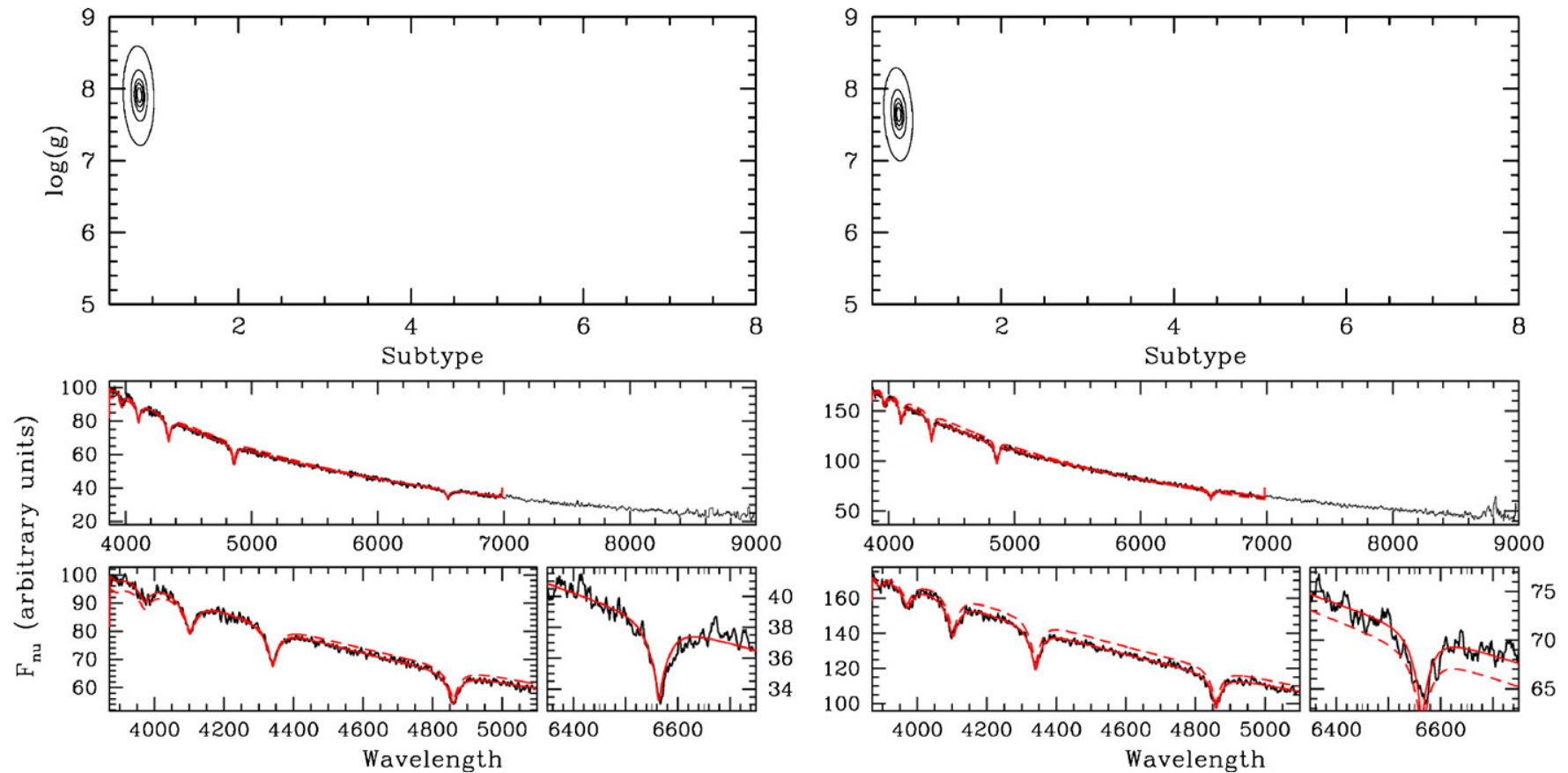

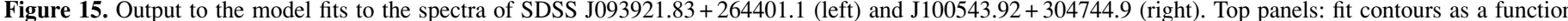

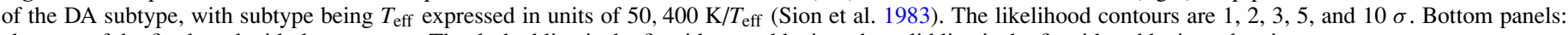
closeups of the fit plotted with the spectrum. The dashed line is the fit without reddening; the solid line is the fit with reddening taken into account.

(A color version of this figure is available in the online journal.)

that were not included in the Kleinman et al. (2004) DR1 or Eisenstein et al. (2006) samples; we refer to all of these objects as post-DR4.

Among the 43 RASS sources with new candidate WDs within $1^{\prime}$, we selected the super-soft sources (HR1 $\leqslant-0.8$ ). Ten sources met this criterion. Two are known DA WDs, WD $1325+512$ and WD $1415+132$ (McCook \& Sion 1999), and known X-ray emitters. ${ }^{31}$ The SDSS spectra indicate that both have M-star companions, and we include these WDs in Table 6.

In two other cases, the SDSS data indicate that another object may be the RASS counterpart. For SDSS $\mathrm{J} 114142.84+143356.5$, a $g=15.76$ star is nearer to the position of the RASS source (1RXS J14145.3+143349). This star, were it to be the X-ray source counterpart, would have $\log \left(f_{X} / f_{g}\right)=-1.04$, which is high for a main-sequence stellar X-ray emitter. On the other hand, this is the least soft of the RASS sources (HR1 $=-0.88 \pm 0.20)$. For SDSS $\mathrm{J} 155109.55+454252.2$, a UV-excess object $(g=17.55, u-g=$ $-0.39 \pm 0.03)$ is virtually coincident with the RASS source (1RXS J155108.6+454312). Since the RASS source is supersoft, this UV-excess object may be a WD or a CV; the latter are also frequently super-soft emitters.

In the remaining six cases, the association of RASS source and SDSS candidate WD is very strong. Fitting the SDSS spectra using WD models then reveals that three of these candidate WDs are in fact hot stars: SDSS J125338.50+051542.1, $\mathrm{J} 133024.24-001636.8$, and J161614.02 + 080905.8.

The other three stars are new DA WDs and included in Table 5: SDSS J093921.83 + 264401.1, J100543.92 + 304744.9,

31 WD $1415+132$ is included in Fleming et al. (1996); WD $1325+512$ is listed in SIMBAD as a known X-ray source, but is not included in Fleming et al. (1996) or Zickgraf et al. (2003). It is unclear how it was identified as a RASS source. and $\mathrm{J} 143736.67+362213.4$. Because of calibration problems with the SDSS data, the fitting failed to return values for $T_{\text {eff }}$ and $\log g$ for the spectrum of SDSS J143736.67+ 362213.4 (the spectrum is also truncated at $7400 \AA$ ). The fits for the two other DAs are shown in Figure 15.

\section{REFERENCES}

Abazajian, K., et al. 2003, AJ, 126, 2081

Adelman-McCarthy, J. K., et al. 2006, ApJS, 162, 38

Adelman-McCarthy, J. K., et al. 2008, ApJS, 175, 297

Agüeros, M. A. 2006, PhD Thesis, Univ. Washington, Washington

Agüeros, M. A., et al. 2006, AJ, 131, 1740

Anderson, S. F., et al. 2003, AJ, 126, 2209

Anderson, S. F., et al. 2007, AJ, 133, 313

Antiochos, S. K., et al. 1986, ApJ, 307, L55

Appenzeller, I., et al. 1998, ApJS, 117, 319

Ayres, T. R., et al. 1981, ApJ, 250, 293

Bade, N., et al. 1998, A\&AS, 127, 145

Baldwin, J. A., Phillips, M. M., \& Terlevich, R. 1981, PASP, 93, 5

Barstow, M. A. 1989, IAU Colloq. 114: White Dwarfs, Lecture Notes in Phys. Vol. 328 (Dordrecht: Kluwer), 156

Bauer, F. E., et al. 2000, ApJS, 129, 547

Becker, R. H., et al. 1995, ApJ, 450, 559

Bowyer, S., Lampton, M., Lewis, J., Wu, X., Jelinsky, P., \& Malina, R. F. 1996, ApJS, 102, 129

Cassinelli, J. P., \& Swank, J. H. 1983, ApJ, 271, 681

Chu, Y.-H., et al. 2004, AJ, 127, 477

Cohen, M., Wheaton, W. A., \& Megeath, S. T. 2003, AJ, 126, 1090

Comastri, A., et al. 2002, ApJ, 571, 771

Condon, J. J., et al. 1998, AJ, 115, 1693

Covey, K. R., et al. 2007, AJ, 134, 2398

Covey, K. R., et al. 2008, ApJS, 178, 339

Cutri, R. M., et al. 2003, 2MASS All Sky Catalog of Point Sources (The IRSA 2MASS All-Sky Point Source Catalog NASA/IPAC Infrared Science Archive, http://irsa.ipac.caltech.edu/applications/Gator/)

Daniel, K. J., et al. 2002, ApJ, 578, 486 
Dempsey, R. C., Linsky, J. L., Fleming, T. A., \& Schmitt, J. H. M. M. 1993, ApJS, 86,599

Drake, S. A., Simon, T., \& Linsky, J. L. 1992, ApJS, 82, 311

Eisenstein, D. J., et al. 2006, ApJS, 167, 40

Farihi, J., Hoard, D. W., \& Wachter, S. 2006, ApJ, 646, 480

Favata, F., Barbera, M., Micela, G., \& Sciortino, S. 1995, A\&A, 295, 147

Feigelson, E. D., et al. 2004, ApJ, 611, 1107

Finlator, K., et al. 2000, AJ, 120, 2615

Fischer, J.-U., Hasinger, G., Schwope, A. D., Brunner, H., Boller, T., Trümper, J., Voges, W., \& Neizvestnyj, S. 1998, Astron. Nachr., 319, 347

Fleming, T. A. 1998, ApJ, 504, 461

Fleming, T. A., Gioia, I. M., \& Maccacaro, T. 1989a, ApJ, 340, 1011

Fleming, T. A., Gioia, I. M., \& Maccacaro, T. 1989b, AJ, 98, 692

Fleming, T. A., et al. 1996, A\&A, 316, 147

Flesch, E., \& Hardcastle, M. J. 2004, A\&A, 427, 387

Fukugita, M., et al. 1996, AJ, 111, 1748

Gioia, I. M., et al. 1984, ApJ, 283, 495

Gioia, I. M., et al. 1990, ApJS, 72, 567

Gioia, I. M., et al. 2003, ApJS, 149, 29

Giommi, P., et al. 1991, ApJ, 378, 77

Güdel, M. 2004, A\&AR, 12, 71

Gunn, J. E., et al. 1998, AJ, 116, 3040

Gunn, J. E., et al. 2006, AJ, 131, 2332

Hagen, H.-J., et al. 1995, A\&AS, 111, 195

Haisch, B., \& Schmitt, J. H. M. M. 1994, ApJ, 426, 716

Haisch, B., et al. 1991, ApJ, 383, L15

Harnden, F. R., et al. 1979, ApJ, 234, L51

Hawley, S. L., \& Pettersen, B. R. 1991, ApJ, 378, 725

Hempelmann, A., Schmitt, J. H. M. M., \& Stępień, K. 1996, A\&A, 305, 284

Høg, E., et al. 1998, A\&A, 335, L65

Hogg, D. W., et al. 2001, AJ, 122, 2129

Homer, L., et al. 2006, AJ, 132, 2743

Hünsch, M., Schmitt, J. H. M. M., Sterzik, M. F., \& Voges, W. 1999, A\&AS, 135,319

Hünsch, M., Schmitt, J. H. M. M., \& Voges, W. 1998a, A\&AS, 127, 251

Hünsch, M., et al. 1998b, A\&AS, 132, 155

Hussain, G. A. J., et al. 2007, MNRAS, 377, 1488

Ivezić, Ž., et al. 2004, Astron. Nachr., 325, 583

Kleinman, S. J., et al. 2004, ApJ, 607, 426

Kraus, A. L., \& Hillenbrand, L. A. 2007, AJ, 134, 2340

Krautter, J., et al. 1999, A\&A, 350, 743

López-Corredoira, M., Gutiérrez, C. M., Mohan, V., Gunthardt, G. I., \& Alonso, M. S. 2008, A\&A, 480, 61

Maccacaro, T., et al. 1988, ApJ, 326, 680

Marsh, M. C., et al. 1997, MNRAS, 286, 369

McCook, G. P., \& Sion, E. M. 1999, ApJS, 121, 1

McGlynn, T. A., et al. 2004, ApJ, 616, 1284

Metanomski, A. D. F., Pasquini, L., Krautter, J., Cutispoto, G., \& Fleming, T. A. 1998, A\&AS, 131, 197

Mickaelian, A. M., et al. 2006, A\&A, 449, 425

Monet, D., et al. 1998, VizieR Online Data Catalog, 1252, 0

Motch, C., et al. 1998, A\&AS, 132, 341

Nieto-Santisteban, M. A., et al. 2004, in ASP Conf. Ser. 314, Astronomical Data Analysis Software and Systems (ADASS) XIII, ed. F. Ochsenbein, M. G. Allen, \& D. Egret (San Francisco, CA: ASP), 666

O’Dwyer, I. J., et al. 2003, AJ, 125, 2239
Osten, R. A., et al. 2005, ApJ, 621, 398

Parejko, J. K., Constantin, A., Vogeley, M. S., \& Hoyle, F. 2008, AJ, 135, 10

Patterson, J., \& Raymond, J. C. 1985, ApJ, 292, 535

Pier, J. R., et al. 2003, AJ, 125, 1559

Plotkin, R. M., Anderson, S. F., Hall, P. B., Margon, B., Voges, W., Schneider, D. P., Stinson, G., \& York, D. G. 2008, AJ, 135, 2453

Reid, I. N., \& Hawley, S. L. 2005, New Light on Dark Stars: Red Dwarfs, Low-Mass Stars, Brown Dwarfs, Springer-Praxis Books in Astrophysics and Astronomy, ed. I. N. Reid \& S. L. Hawley (Chichester: Praxis Publishing Ltd.)

Richards, G. T., et al. 2002, AJ, 123, 2945

Richman, H. R. 1996, ApJ, 462, 404

Rogel, A. B., et al. 2006, ApJS, 163, 160

Rutledge, R. E., et al. 2000, ApJS, 131, 335

Schmidt, G. D., et al. 2005a, ApJ, 630, 1037

Schmidt, G. D., et al. 2005b, ApJ, 620, 422

Schmitt, J. H. M. M., \& Liefke, C. 2004, VizieR Online Data Catalog, 341, 70651

Schmitt, J. H. M. M., et al. 1995, ApJ, 450, 392

Schreier, E., et al. 1972, ApJ, 172, L79

Schröder, C., \& Schmitt, J. H. M. M. 2007, A\&A, 475, 677

Schwope, A., et al. 2000, Astron. Nachr., 321, 1

Scranton, R., et al. 2002, ApJ, 579, 48

Shaw, J. S., Caillault, J.-P., \& Schmitt, J. H. M. M. 1996, ApJ, 461, 951

Shipman, H. L. 1976, ApJ, 206, L67

Silvestri, N. M., et al. 2006, AJ, 131, 1674

Simon, T., Drake, S. A., \& Kim, P. D. 1995, PASP, 107, 1034

Sion, E. M., et al. 1983, ApJ, 269, 253

Skrutskie, M. F., et al. 2006, AJ, 131, 1163

Smith, J. A., et al. 2002, AJ, 123, 2121

Smith, K., et al. 2005, A\&A, 436, 241

Stephenson, C. B. 1986, AJ, 91, 144

Stocke, J. T., et al. 1983, ApJ, 273, 458

Stocke, J. T., et al. 1991, ApJS, 76, 813

Stoughton, C., et al. 2002, AJ, 123, 485

Szkody, P., et al. 2002, AJ, 123, 430

Szkody, P., et al. 2003, AJ, 126, 1499

Szkody, P., et al. 2004a, AJ, 128, 1882

Szkody, P., et al. 2004b, AJ, 128, 2443

Szkody, P., et al. 2005, AJ, 129, 2386

Szkody, P., et al. 2006, AJ, 131, 973

Szkody, P., et al. 2007, AJ, 134, 185

Tananbaum, H., et al. 1972, ApJ, 174, L143

Topka, K., Golub, L., Gorenstein, P., Harnden, F. R., Jr., Vaiana, G. S., Avni, Y., \& Rosner, R. 1982, ApJ, 259, 677

Tucker, D. L., et al. 2006, Astron. Nachr., 327, 821

Voges, W., et al. 1999, A\&A, 349, 389

Voges, W., et al. 2000, VizieR Online Data Catalog, 9029, 0

Wachter, S., et al. 2003, ApJ, 586, 1356

Walter, F. M., Cash, W., Charles, P. A., \& Bowyer, C. S. 1980, ApJ, 236, 212

Woudt, P. A., et al. 2004, MNRAS, 351, 1015

York, D. G., et al. 2000, AJ, 120, 1579

Zickgraf, F.-J., Krautter, J., Reffert, S., Alcalá, J. M., Mujica, R., Covino, E., \& Sterzik, M. F. 2005, A\&A, 433, 151

Zickgraf, F.-J., et al. 1997, A\&AS, 123, 103

Zickgraf, F.-J., et al. 2003, A\&A, 406, 535 